OPEN ACCESS

Edited by:

Giancarlo Condello,

University of Parma, Italy

Reviewed by:

Umer Asgher,

National University of Sciences and Technology, Pakistan

Atsuhiro Tsubaki,

Niigata University of Health and Welfare, Japan

*Correspondence:

Bart Roelands

Bart.Roelands@vub.be

Specialty section: This article was submitted to

Exercise Physiology,

a section of the journa

Frontiers in Physiology

Received: 19 August 2021 Accepted: 27 September 2021

Published: 26 October 2021

Citation:

De Wachter J, Proost M, Habay J, Verstraelen M, Díaz-García J, Hurst P,

Meeusen $R$, Van Cutsem $J$ and Roelands B (2021) Prefrontal Cortex

Oxygenation During Endurance Performance: A Systematic Review of Functional Near-Infrared Spectroscopy Studies.

Front. Physiol. 12:761232. doi: 10.3389/fphys.2021.761232

\section{Prefrontal Cortex Oxygenation During Endurance Performance: A Systematic Review of Functional Near-Infrared Spectroscopy Studies}

\author{
Jonas De Wachter ${ }^{1}$, Matthias Proost ${ }^{1}$, Jelle Habay ${ }^{1}$, Matthias Verstraelen', \\ Jesús Díaz-García ${ }^{2}$, Philip Hurst ${ }^{3}$, Romain Meeusen ${ }^{1}$, Jeroen Van Cutsem ${ }^{1,4}$ and \\ Bart Roelands ${ }^{1 *}$
}

\begin{abstract}
${ }^{1}$ Human Physiology and Sports Physiotherapy Research Group, Vrije Universiteit Brussel, Brussels, Belgium, ${ }^{2}$ Faculty of Sport Sciences, University of Extremadura, Caceres, Spain, ${ }^{3}$ The School of Psychology \& Life Sciences, Canterbury Christ Church University, Canterbury, United Kingdom, ${ }^{4}$ VIPER Research Unit, Royal Military Academy, Brussels, Belgium
\end{abstract}

Introduction: A myriad of factors underlie pacing-/exhaustion-decisions that are made during whole-body endurance performance. The prefrontal cortex (PFC) is a brain region that is crucial for decision-making, planning, and attention. PFC oxygenation seems to be a mediating factor of performance decisions during endurance performance. Nowadays, there is no general overview summarizing the current knowledge on how PFC oxygenation evolves during whole-body endurance performance and whether this is a determining factor.

Methods: Three electronic databases were searched for studies related to the assessment of PFC oxygenation, through near-IR spectroscopy (NIRS), during endurance exercise. To express PFC oxygenation, oxygenated $\left(\mathrm{HbO}_{2}\right)$ and deoxygenated hemoglobin $(\mathrm{HHb})$ concentrations were the primary outcome measures.

Results: Twenty-eight articles were included. Ten articles focused on assessing prefrontal oxygenation through a maximal incremental test (MIT) and 18 focused on using endurance tasks at workloads ranging from low intensity to supramaximal intensity. In four MIT studies measuring $\mathrm{HbO}_{2}$, an increase of $\mathrm{HbO}_{2}$ was noticed at the respiratory compensation point $(\mathrm{RCP})$, after which it decreased. $\mathrm{HbO}_{2}$ reached a steady state in the four studies and increased in one study until exhaustion. All studies found a decrease or steady state in $\mathrm{HHb}$ from the start until $\mathrm{RCP}$ and an increase to exhaustion. In regard to (non-incremental) endurance tasks, a general increase in PFC oxygenation was found while achieving a steady state at vigorous intensities. PCF deoxygenation was evident for near-to-maximal intensities at which an increase in oxygenation and the maintenance of a steady state could not be retained.

Discussion/Conclusion: MIT studies show the presence of a cerebral oxygenation threshold (ThCox) at RCP. PFC oxygenation increases until the RCP threshold, thereafter, a steady state is reached and $\mathrm{HbO}_{2}$ declines. This study shows that the results obtained from MIT are transferable to non-incremental endurance exercise. $\mathrm{HbO}_{2}$ 
increases during low-intensity and moderate-intensity until vigorous-intensity exercise, and it reaches a steady state in vigorous-intensity exercise. Furthermore, ThCox can be found between vigorous and near-maximal intensities. During endurance exercise at near-maximal intensities, PFC oxygenation increases until the value exceeding this threshold, resulting in a decrease in PFC oxygenation. Future research should aim at maintaining and improving PFC oxygenation to help in improving endurance performance and to examine whether PFC oxygenation has a role in other performancelimiting factors.

Keywords: near-infrared spectroscopy (NIRS), endurance exercise, prefrontal cortex, oxygenation, respiratory compensation point (RCP), systematic review

\section{INTRODUCTION}

Dynamic endurance exercise can be defined as prolonged ( $>75 \mathrm{~s}$ ) exercise and can be classified into whole-body endurance and local muscle endurance (Mccormick et al., 2015). Dynamic whole-body endurance exercise involves large muscle groups (e.g., cycling, running, and rowing), whereas muscle endurance exercise involves only one muscle or muscle group (e.g., knee extension or handgrip tasks) (Kenney et al., 2012; Pageaux et al., 2013; Mccormick et al., 2015). In general, whole-body endurance tasks are most frequently measured during time-to-exhaustion (TTE), time-trials (TTs) (Amann et al., 2008), and constant intensity fixed duration (CIFD) tasks (i.e., both intensity and duration are fixed factors) (Ichinose et al., 2020). In contrast to TT and TTE performance, CIFD tasks are mainly used to assess psychological (e.g., perceived exertion rating, thermal discomfort, etc.) and physiological (e.g., heart rate (HR), blood lactate, etc.) reactions during exercise.

During both TTE and TTs, important decisions that impact performance need to be taken into consideration. In TTE, participants need to decide when to stop (e.g., "Am I totally exhausted and have to stop this exercise?") and in TTs, participants need to decide how much effort to give (e.g., "When should I slow down/speed up to reach the set goal as fast/good as possible?"). A large body of research has examined the underlying mechanisms of these decisions. Périard and Racinais (2015) suggested that decisions during TTs in hypoxic- and hot conditions are related to oxygen availability, whereas Girard and Racinais (2014) reported that decisions during TTE are related to alterations in central command. Similarly, core temperatures exceeding $>39^{\circ} \mathrm{C}$ and oxygen saturation dropping to less than $<70 \% \mathrm{O}_{2}$ saturation have been associated with performance-related decisions in both TT and TTE (Racinais and Girard, 2012). Psychobiological factors are also likely to play a role. Van Cutsem et al. (2019) reported an increase in subjective thermal strain resulted in a decrease in performance (i.e., an earlier exhaustion-decision). Given this evidence, it is clear that a myriad of factors plays a role in the underlying mechanisms of decisions in both TTE and TTs.

All the proposed mediating factors that play a role in decisions in TTE and TTs are likely to be located both peripherally (i.e., muscles) and centrally (i.e., the brain and central nervous system). Peripherally, for example, locomotor muscle fatigue is mediated by the accumulation of intracellular metabolites, which eventually cause failure in excitationcontraction coupling (Amann, 2011). Whereas centrally, it is theoretically hypothesized that the corollary discharge model determines the perception of effort by sending a copy of a motor command to the somatosensory areas. These corollary discharges influence performance decisions about how much effort is needed to give and highlight the brain as an important mediating factor in decision-making in both TTs and TTE.

The top-downregulation of the prefrontal cortex (PFC) during exercise tolerance and volition is also likely to play a role in performance decisions (Robertson and Marino, 2016). After applying 30-min transcranial direct current stimulation to the dorsolateral PFC, Angius et al. (2019) reported improvements in TTE, lower ratings of perceived exertion (RPE) and HR, and higher blood lactate than no-treatment controls. Angius et al. (2019) concluded that by targeting PFC using transcranial direct current stimulation, improvements in performance were the result of a change in decision-making, planning, attention, (short-term) memory, and executive function. A body of research has shown that PFC is an underlying mechanism for the termination of whole-body endurance exercise and is mediated by the oxygenation of the cerebral cortex (Ide et al., 1999; Ide and Secher, 2000; Bhambhani et al., 2007; Rupp and Perrey, 2008; Rooks et al., 2010). It is likely that PFC oxygenation during incremental exercise increases from low-to-hard intensities, and declines to preceding exhaustion (Rooks et al., 2010).

As mentioned earlier, PFC oxygenation seems to be an important and promising mediating factor of performance decisions during whole-body endurance performance. The two techniques that can be used to assess PFC oxygenation are functional MRI (fMRI) and functional near-IR spectroscopy (fNIRS). fMRI is suggested to be the gold standard for measuring changes in brain oxygenation and provides a high spatial resolution (up to $4.0 \mathrm{~mm}$ ) that, in turn, can help to measure functional hemodynamic changes in the brain (Glover, 2011; Herold et al., 2018). On the other hand, fMRI is expensive and results in relatively low temporal resolution $(\approx 0.5 \mathrm{~Hz})$ due to its sensitivity to the movement (Ekkekakis, 2009; Herold et al., 2018). fNIRS is a noninvasive optical imaging technique that measures relative 
changes in hemoglobin $(\mathrm{Hb})$ concentrations. Compared to fMRI, fNIRS provides researchers with more possibilities to examine performance in ecological settings given that it is less sensitive to movement. However, the spatial resolution for fNIRS $(\approx 10-20 \mathrm{~mm}$ ) is inferior to that for fMRI (Glover, 2011; Scarapicchia et al., 2017). This disadvantage has, however, not held back researchers from attempting to create further insights into the role of (prefrontal) brain oxygenation in performance-related decisions during whole-body endurance performance (e.g., Rooks et al., 2010; Leff et al., 2011). Rooks et al. (2010) reported that PFC oxygenation during incremental exercise increased between moderate and hard intensities after which it drastically decreased at very hard intensities. Leff et al. (2011) replicated these results and reported that cortical oxygenation increased during the first few minutes following exercise until near-maximal exercise and a decrease in PFC oxygenation at near-maximal to intense exhaustive exercise. Herold et al. (2018) summarized the methodological knowledge of fNIRS, outlined recommendations for future research (Herold et al., 2018), and confirmed the relevance and applicability of fNIRS during exercise science. However, it has been over a decade since Rooks et al. (2010) summarized the effects of incremental exercise on cerebral oxygenation. To date, there is no general overview summarizing current knowledge on how PFC oxygenation evolves during (non-incremental) whole-body endurance exercise at different intensities and whether this influences performance. Therefore, there is a need for a stateof-the-art summary of cerebral oxygenation during whole-body endurance performance.

As mentioned earlier, it is clear that fNIRS research can help elucidate the underlying mechanisms of performancerelated decisions during endurance exercise. To advance the field of whole-body endurance performance and highlight the opportunities to evaluate the impact of specific countermeasures on the performance-related changes in fNIRS variables, the aim of this systematic review is to provide an updated review of the extant research. We hypothesize that: (1) prefrontal oxygenation increases during submaximal exercise and subsequently decreases at a near-maximal intensity, (2) prefrontal oxygenation increases during prolonged submaximal exercise until a steady state is reached, and (3) at near-maximal intensities PFC oxygenation cannot be maintained, resulting in a decrease near exhaustion.

\section{METHODS}

This systematic review followed the guidelines provided by the Preferred Reporting Items for Systematic Review and MetaAnalyses statement (Page et al., 2021).

\section{Eligibility Criteria}

To determine keywords for a review, we used the participants, intervention, comparison, outcome, and study design (PICOS) search tool (see Table 1). Studies with a healthy human adult population, aged between 18 and 45 years old, and without gender restrictions were included. Given that an aerobic energy
TABLE 1 | "PICOS" categories (participants, interventions, comparisons, outcomes, study design) used to determine keywords.

\begin{tabular}{ll}
\hline PICOS component & Detail \\
\hline $\begin{array}{l}\text { Participants } \\
\text { Interventions }\end{array}$ & $\begin{array}{l}\text { Healthy humans (18-45 years) } \\
\text { Whole-body endurance performance task (>75 s) } \\
\text { Baseline NIRS-measures before the start of and/or } \\
\text { during the whole-body endurance performance task or } \\
\text { the control condition (e.g., exercise modality) }\end{array}$ \\
Outcomes & $\begin{array}{l}\text { Prefrontal cortex oxygenation (NIRS) during whole-body } \\
\text { endurance task or performance }\end{array}$ \\
Study designs & RCTs, nRCT, nRnCTs
\end{tabular}

NIRS, Near infrared spectroscopy RCT randomized controlled trial; nRCT nonrandomized controlled trials; $n R n C T s$ non-randomized non-controlled trials.

system predominates during maximum effort exercise after 75 s (Gastin, 2001), we included the studies using dynamic whole-body endurance performance with a duration of at least 75 s. Maximal incremental exercise tasks were also included in this review. The two main outcome parameters for inclusion were performance on the exercise task and PFC oxygenation. A baseline measure at the start of the exercisetask had to be made for cerebral oxygenation to be able to evaluate the evolution of oxygenation throughout the trials. Randomized controlled trials (RCTs), non-RCTs (nRCTs), or non-randomized non-controlled trials (nRnCTs), written in English, were considered to be eligible for inclusion. Studies were excluded when the study population was patients who were using medications. Additionally, animal studies, individual case studies, and interventions where participants performed with their eyes closed were also excluded.

\section{Information Sources and Search Strategy}

Three electronic databases, PubMed, Web of Science, and Cochrane Library (until August 20, 2021), were searched with no date restrictions. Medical subject heading (mesh) terms, if available in PubMed and Cochrane Library, were used for a qualitative literature search. The following keywords were applied individually or in combination: Near Infrared Spectroscopy, NIRS, Magnetic Resonance Imaging, MRI, Near Infrared Spectroscopy [MeSH], Magnetic Resonance Imaging $[\mathrm{MeSH}]$, Physical performance, Exercise performance, Endurance performance, Time trial, Time to exhaustion, physical endurance, Athletic performance, physical endurance $[\mathrm{MeSH}]$, Athletic performance $[\mathrm{MeSH}]$, Motor Activity $[\mathrm{MeSH}]$, Oxygenation, BOLD response, hemodynamic response, Cerebrovascular Circulation, Cerebrovascular Circulation [MeSH] prefrontal cortex OR frontal lobe OR frontal lobe [MeSH] (Table 2). The combination of keywords 1, 2, and 3 (see Table 2) was included in a search strategy. In addition, to ensure the literature saturation, a backward search and a forward search were performed by screening the reference lists of the included articles and by screening the citations of the included studies, respectively, to increase the likelihood of the inclusion of all relevant studies. 
TABLE 2 | Search strategy: number of hits on keywords and combined keywords in PubMed, Web of Science, and Cochrane Library.

\begin{tabular}{|c|c|c|c|}
\hline \multirow[t]{2}{*}{ Keywords } & PubMed & Web of science & Cochrane library \\
\hline & $\begin{array}{l}\text { No. of hits } \\
(20 / 08 / 2020)\end{array}$ & $\begin{array}{l}\text { No. of hits } \\
(20 / 08 / 2020)\end{array}$ & $\begin{array}{l}\text { No. of hits } \\
(20 / 08 / 2020)\end{array}$ \\
\hline $\begin{array}{l}\text { (1) Near infrared spectroscopy OR NIRS OR magnetic resonance imaging OR MRI or near } \\
\text { infrared spectroscopy [MeSH] OR magnetic resonance imaging [MeSH] }\end{array}$ & 608,218 & 487,775 & 33,168 \\
\hline $\begin{array}{l}\text { (2) Physical performance OR exercise performance OR endurance performance OR time } \\
\text { trial OR time to exhaustion OR physical endurance OR athletic performance OR physical } \\
\text { endurance [MeSH] OR athletic performance [MeSH] OR motor activity [MeSH] }\end{array}$ & 336,117 & 137,120 & 31,398 \\
\hline $\begin{array}{l}\text { (3) Oxygenation OR BOLD response OR hemodynamic response OR cerebrovascular } \\
\text { circulation OR cerebrovascular circulation }[\mathrm{MeSH}]\end{array}$ & 124,655 & 77,973 & 51,012 \\
\hline (4) prefrontal cortex OR frontal lobe OR frontal lobe [MeSH] & 107,239 & 118,480 & 7524 \\
\hline \multicolumn{4}{|l|}{ Combined key words } \\
\hline (1) AND (2) & 6566 & 549 & 1664 \\
\hline (1) AND (2) AND (3) & 4650 & 389 & 352 \\
\hline (1) AND (2) AND (3) AND (4)* & 921 & 202 & 335 \\
\hline (1) AND (2) AND (4) & 1408 & 48 & 350 \\
\hline
\end{tabular}

Combined keywords with a "**" were included in the study.

\section{Study Selection and Data Collection Process}

Articles from the three databases were collated in EndNote X9 where the duplicates were removed (Table 3). Afterward, all studies were imported into Rayyan (the web and mobile app for systematic reviewers; Ouzzani et al., 2016), where the two reviewers (JDW and JH), independently and blinded from each other, screened the title and abstracts for each study. The search resulted in 89 (7\%) conflicting studies. After the conflicts were resolved, the full text of the remaining articles was screened. A general meeting with the research team was held to decide on inclusion. The full-text version of all the articles that met the inclusion criteria (Table 3) was retrieved for quality assessment and data extraction (see quality assessment). If, after this screening, a citation was considered potentially eligible for inclusion and relevant, the full-text article was retrieved. A flow diagram illustrating the selection of the included studies can be found in Figure 1.

\section{Quality Assessment}

Methodological quality was assessed using the quantitative assessment tool "QualSyst" (Kmet and Lee, 2004). The QualSyst tool is a checklist containing 14 items, which are plotted on a 3point scale (yes $=2$, partial $=1$, and no $=0$ ). Items that were not applicable to a particular study design were marked as " $\mathrm{n} / \mathrm{a}$ " and were excluded from the calculation of a summary score (Kmet and Lee, 2004). A quality score was calculated for each paper by summing the total score obtained across relevant items and dividing them by the total possible score (Kmet and Lee, 2004). The two reviewers (JDW and JH) independently performed quality assessments, and disagreements were solved through a consensus. A score of $\geq 75 \%, 55-75 \%$, and $\leq 55 \%$ indicated strong quality, moderate quality, and weak quality, respectively.

\section{Classification of Intensity}

Based on the ACSM position stand, the classification of exercise intensity: relative and absolute exercise intensity for
TABLE 3 | Inclusion and exclusion criteria.

\begin{tabular}{ll}
\hline Inclusion & Exclusion \\
\hline fNIRS & Individual case studies \\
Healthy participants (18-45 years) & Animal studies \\
Neutral ambient conditions & Eyes closed \\
Cycling/running/rowing & Psychiatric disorders/patients \\
Whole-body endurance performance task & Medication \\
\hline
\end{tabular}

fNIRS, functional Near-Infrared Spectroscopy.

cardiorespiratory endurance and resistance exercise, whole-body endurance performance tasks were classified into very light, light, moderate, vigorous, and near-maximal intensities (Garber et al., 2011). The classification, where necessary, was based on energy cost calculations using the ACSM energy equation for cycling (Swain, 2000).

\section{RESULTS}

\section{Study Selection}

After the removal of duplicates, the search strategy resulted in 1,260 articles. After screening the titles and abstracts of the remaining 54 (Figure 1), 25 met the inclusion criteria. After screening the reference lists of the full-text articles additional three articles were included, resulting in a total of 28 articles, of which $53 \%$ were scored as strong, $36 \%$ as moderate, and only $11 \%$ as weak on the quality assessment (as shown in Table 4).

\section{Characteristics of Near-IR Spectroscopy}

Positioning of the optodes and receivers with the associated interoptode distances (IODs) varies and is described in Tables 5A,B. Nineteen studies used the international EEG 10-20 system with optode placements over the prefrontal lobe at Fp1,Fp2, Fp3, and/or Fp4 position (Rupp and Perrey, 2008; Timinkul et al., 2008; Billaut et al., 2010; Keramidas et al., 2011; Rupp et al., 2013; Giles et al., 2014; Oussaidene et al., 2015; 
Identification of studies via databases and registers

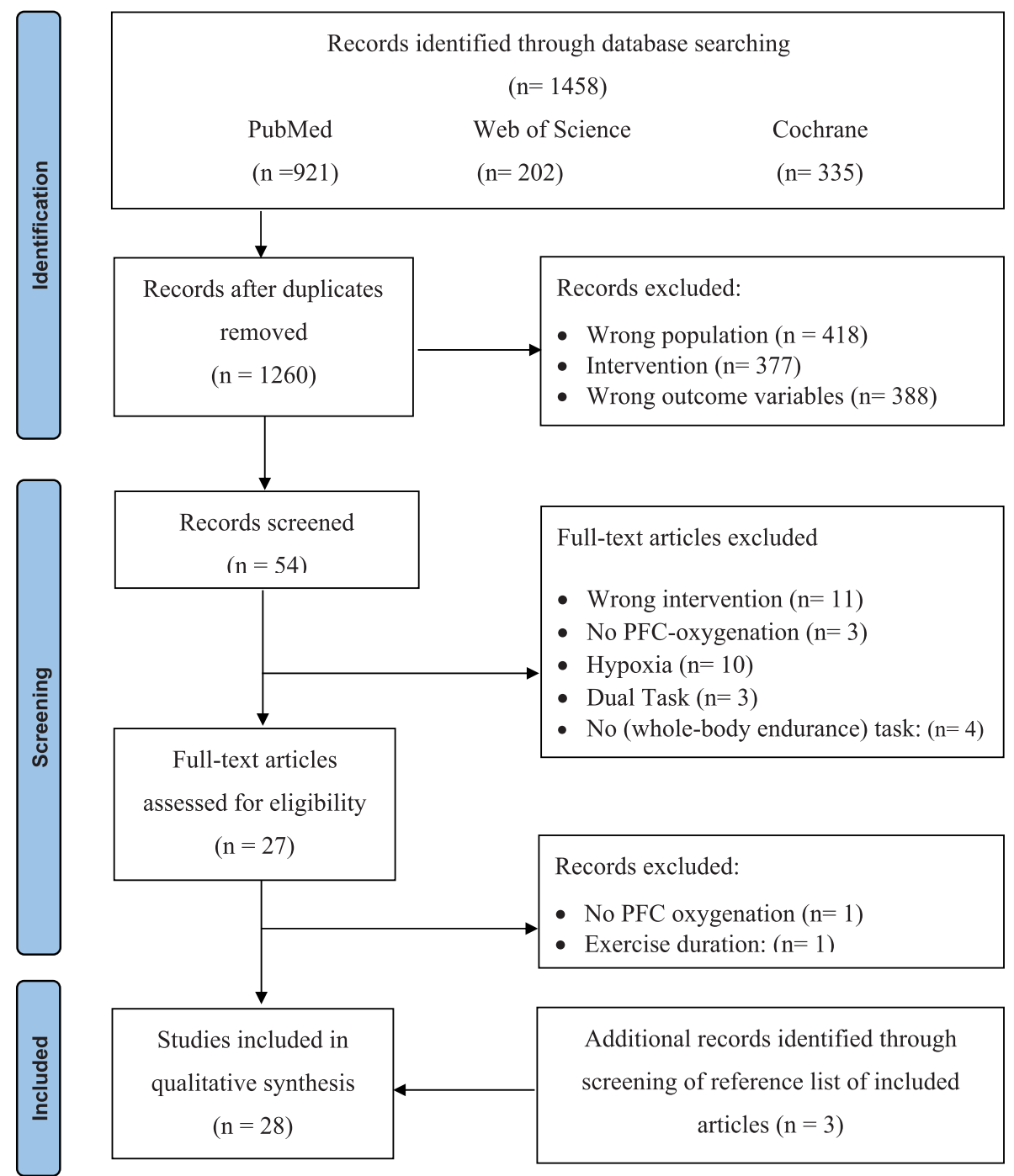

FIGURE 1 | Selection process for research articles $(n=28)$ included in this systematic review. Adapted version of the recommendations in the PRISMA 2020 statement (Liberati et al., 2009). $n R C T$, non-randomized controlled trial; $n R n C T$, non-randomized non-controlled trial; $R C T$, randomized controlled trial.

Santos-Concejero et al., 2015, 2017; Pires et al., 2016; Tempest and Parfitt, 2016; Tsubaki et al., 2016, 2018, 2020; Takehara et al., 2017; Tempest et al., 2017; Asahara and Matsukawa, 2018; Ohyanagi et al., 2018; Stevens et al., 2018) pecifying this location as $\pm 3 \mathrm{~cm}$ from the midline, just above the supra-orbital ridge (Bhambhani et al., 2007; Miyazawa et al., 2013). Jung et al. (2015) used Broadman area 10, Montreal Neurophysiological Institute $(\mathrm{MNI})$ coordinates $[(x / y / z)-40$, $50,0]$, two studies performed a three-dimensional T1-weighted MRI scan, marking the optode location for the left and right PFC (Suzuki et al., 2004; Fumoto et al., 2010), and finally, two studies placed the device on the participants' forehead without specifying the exact location of the optode placement (Shibuya et al., 2004; Kounalakis and Geladas, 2012).
The IODs varied between 25, 30, 38, 40, 45, and $50 \mathrm{~mm}$ (mean \pm SD: $38 \pm 7 \mathrm{~mm}$ ).

\section{Prefrontal Cortex Oxygenation During Maximal Incremental Exercise}

Eleven of the included studies evaluated the effects of exercise intensity on cerebral cortex oxygenation using an incremental cycling protocol until exhaustion (Bhambhani et al., 2007; Rupp and Perrey, 2008; Timinkul et al., 2008; Tempest et al., 2014, 2017; Jung et al., 2015; Oussaidene et al., 2015; Tempest and Parfitt, 2016; Tsubaki et al., 2016; Stevens et al., 2018; Kojima et al., 2021). Incremental exercise protocols were characterized by systematic increases in intensity over time. The protocols 
TABLE 4 | Quality assessment “QualSyst” (Kmet and Lee, 2004).

\begin{tabular}{|c|c|c|c|c|c|c|c|c|c|c|c|c|c|c|c|}
\hline Study & A & B & C & D & $\mathbf{E}$ & $\mathbf{F}$ & G & $\mathbf{H}$ & I & $\mathbf{J}$ & $\mathbf{K}$ & $\mathbf{L}$ & $\mathbf{M}$ & $\mathbf{N}$ & Rating* \\
\hline Shibuya et al. (2004) & 2 & 2 & 1 & 2 & NA & NA & NA & 2 & 1 & 2 & 2 & NA & 2 & 2 & Strong: 90 \\
\hline Suzuki et al. (2004) & 1 & 1 & 1 & 2 & NA & NA & NA & 1 & 1 & 2 & 1 & NA & 2 & 1 & Moderate: 65 \\
\hline Billaut et al. (2010) & 2 & 2 & 2 & 2 & NA & NA & NA & 1 & 1 & 2 & 2 & NA & 2 & 2 & Strong: 90 \\
\hline Fumoto et al. (2010) & 1 & 1 & 2 & 2 & NA & NA & NA & 1 & 1 & 1 & 2 & NA & 1 & 2 & Moderate: 70 \\
\hline Keramidas et al. (2011) & 2 & 1 & 1 & 2 & 0 & 0 & NA & 1 & 1 & 2 & 2 & 1 & 1 & 2 & Moderate: 62 \\
\hline Miyazawa et al. (2013) & 2 & 1 & 1 & 2 & NA & NA & NA & 1 & 1 & 2 & 2 & NA & 1 & 2 & Strong: 75 \\
\hline Rupp et al. (2013) & 2 & 1 & 1 & 2 & NA & NA & NA & 2 & 1 & 2 & 2 & NA & 1 & 1 & Strong: 75 \\
\hline Giles et al. (2014) & 2 & 1 & 2 & 2 & 0 & 0 & 0 & 2 & 1 & 1 & 1 & 1 & 2 & 2 & Moderate: 61 \\
\hline Santos-Concejero et al. (2015) & 2 & 2 & 2 & 2 & NA & NA & NA & 2 & 1 & 2 & 1 & NA & 1 & 2 & Strong: 85 \\
\hline Pires et al. (2016) & 2 & 2 & 2 & 2 & NA & NA & NA & 2 & 1 & 2 & 2 & NA & 2 & 2 & Strong: 95 \\
\hline Santos-Concejero et al. (2017) & 2 & 1 & 2 & 2 & NA & NA & NA & 2 & 1 & 2 & 2 & NA & 2 & 2 & Strong: 90 \\
\hline Ohyanagi et al. (2018) & 2 & 0 & 0 & 0 & 0 & NA & NA & 1 & 1 & 1 & 2 & 1 & 1 & 2 & Weak: 46 \\
\hline Radel et al. (2017) & 2 & 2 & 2 & 2 & 0 & 1 & 2 & 2 & 2 & 2 & 0 & 1 & 2 & 2 & Strong: 79 \\
\hline Tsubaki et al. (2018) & 2 & 2 & 1 & 1 & NA & NA & NA & 1 & 1 & 2 & 1 & NA & 2 & 1 & Moderate: 70 \\
\hline Kounalakis and Geladas (2012) & 2 & 2 & 1 & 2 & 2 & 0 & 0 & 2 & 1 & 1 & 2 & 2 & 2 & 2 & Strong: 75 \\
\hline Takehara et al. (2017) & 2 & 0 & 1 & 2 & 0 & 0 & 0 & 1 & 1 & 1 & 0 & 1 & 2 & 2 & Weak: 46 \\
\hline Ide et al. (1999) & 1 & 0 & 1 & 2 & NA & NA & NA & 2 & 1 & 1 & 2 & NA & 2 & 1 & Moderate: 65 \\
\hline Bhambhani et al. (2007) & 2 & 2 & 1 & 2 & NA & NA & NA & 2 & 1 & 1 & 2 & NA & 2 & 2 & Strong: 85 \\
\hline Rupp and Perrey (2008) & 2 & 2 & 1 & 2 & NA & NA & NA & 2 & 1 & 2 & 1 & NA & 2 & 2 & Strong: 85 \\
\hline Timinkul et al. (2008) & 2 & 2 & 1 & 2 & NA & NA & NA & 2 & 1 & 2 & 2 & NA & 2 & 2 & Strong: 90 \\
\hline Tempest et al. (2014) & 2 & 2 & 2 & 2 & NA & NA & NA & 2 & 2 & 2 & 2 & NA & 2 & 2 & Strong: 100 \\
\hline Jung et al. (2015) & 2 & 2 & 1 & 0 & NA & NA & NA & 2 & 1 & 1 & 2 & NA & 1 & 1 & Moderate: 65 \\
\hline Oussaidene et al. (2015) & 2 & 2 & 2 & 2 & NA & NA & NA & 2 & 1 & 2 & 2 & NA & 2 & 2 & Strong: 95 \\
\hline Tempest and Parfitt (2016) & 2 & 2 & 2 & 2 & NA & NA & NA & 2 & 2 & 1 & 2 & NA & 2 & 2 & Strong: 95 \\
\hline Tempest et al. (2017) & 2 & 2 & 1 & 1 & 0 & 0 & NA & 2 & 1 & 1 & 2 & 1 & 2 & 2 & Moderate: 65 \\
\hline Stevens et al. (2018) & 1 & 1 & 1 & 1 & 2 & 0 & 1 & 2 & 1 & 2 & 2 & 2 & 2 & 2 & Moderate: 71 \\
\hline Hiura et al. (2018) & 2 & 0 & 1 & 1 & NA & NA & NA & 2 & 1 & 1 & 2 & NA & 2 & 2 & Moderate: 70 \\
\hline Kojima et al. (2020) & 2 & 1 & 1 & 1 & NA & NA & NA & 1 & 0 & 1 & 0 & NA & 0 & 1 & Weak: 40 \\
\hline Kojima et al. (2021) & 2 & 1 & 1 & 1 & NA & NA & NA & 1 & 1 & 1 & 1 & NA & 2 & 1 & Moderate: 60 \\
\hline
\end{tabular}

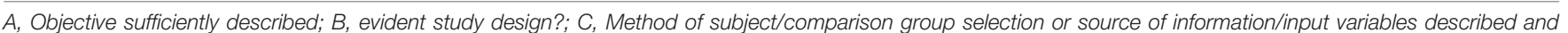

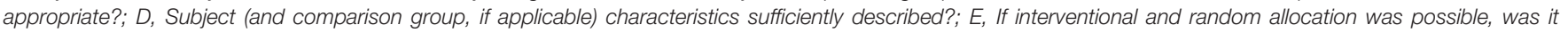

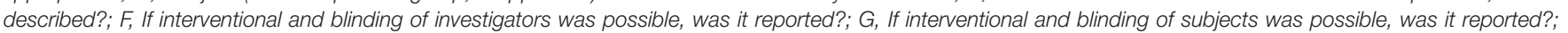

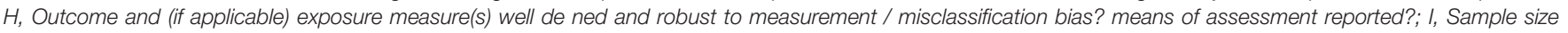

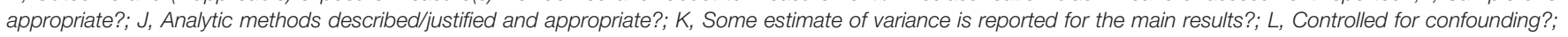
$M$, Results reported in sufficient detail?; N, Results support conclusion?. *Quality scores: $\geq 75 \%$ strong, 55 $\geq 75 \%$ moderate, $\leq 55 \%$ weak.

consisted of fixed increases of $20 \mathrm{~W}$ (Tempest et al., 2014, 2017; Jung et al., 2015; Tempest and Parfitt, 2016; Kojima et al., 2020, 2021), 25 W (Timinkul et al., 2008), and 30 W (Rupp and Perrey, 2008) per $1 \mathrm{~min}, 30 \mathrm{~W}$ per $2 \mathrm{~min}$ (Bhambhani et al., 2007; Stevens et al., 2018), and $1 \mathrm{~W} / \mathrm{kg}$ per $3 \mathrm{~min}$ (Oussaidene et al., 2015) until exhaustion. All protocols started from very low intensities and ranged from no resistance up to $100 \mathrm{~W}$ for the trained athletes. Intensities throughout all the tests were classified as low, moderate, vigorous, and near-maximal to maximal (Garber et al., 2011). From this point, oxygenated $\left(\mathrm{HbO}_{2}\right)$, deoxygenated hemoglobin $(\mathrm{HHb})$, and total hemoglobin $(\mathrm{tHb})$ relate to the PFC unless stated otherwise.

Ten studies reported an increase in $\left[\mathrm{HbO}_{2}\right]$ during the first part of the incremental exercise (Bhambhani et al., 2007; Rupp and Perrey, 2008; Timinkul et al., 2008; Tempest et al., 2014, 2017; Jung et al., 2015; Oussaidene et al., 2015; Stevens et al., 2018; Kojima et al., 2021). Timinkul et al. (2008) described the three distinct phases of prefrontal oxygenation during incremental exercise: (1) the linear-oxygenation phase, where $\mathrm{HbO}_{2}$ gradually increases and $\mathrm{HHb}$ slightly decreases from the start until the so-called cerebral blood volume threshold ( $\left.42 \pm 3.9 \% \mathrm{VO}_{2 \max }\right)$, (2) the hyper-oxygenation phase, where $\mathrm{HbO}_{2}$ rapidly increases until the respiratory compensation point (RCP), while $\mathrm{HHb}$ remains stable, and (3)the desaturation phase, where $\mathrm{HbO}_{2}$ continuously decreases and $\mathrm{HHb}$ increases until exhaustion. However, Timinkul et al. (2008) did not express their results as a percentage of $\mathrm{VO}_{2 \max }$. Similarly, Rupp and Perrey (2008) described an increase in $\left[\mathrm{HbO}_{2}\right]$ from a warm-up to the second ventilatory threshold (VT2, $87.0 \pm 2.0 \% \mathrm{VO}_{2 \max }$ ) and the cerebral oxygenation threshold (ThCox, $86.0 \pm 4.0 \% \mathrm{VO}_{2 \mathrm{max}}$ for untrained and $85.0 \pm 9.0 \% \mathrm{VO}_{2 \max }$ for trained). Similar results were observed for Oussaidene et al. (2015) who reported a decline in $\mathrm{HbO}_{2}$ between VT2 or ThCox and exhaustion. These findings were replicated by Kojima et al. $(2020,2021)$ who reported an increase in $\left[\mathrm{HbO}_{2}\right]$ from the start of exercise until RCP and a subsequent decrease from RCP until exhaustion. Additionally, 
TABLE 5A | Overview of the results within "non-incremental endurance exercise".

\begin{tabular}{|c|c|c|c|c|c|}
\hline Study & $\begin{array}{l}\text { Sample } \\
\text { characteristics } \\
\text { (Mean } \pm \text { SD) }\end{array}$ & $\begin{array}{l}\text { NIRS-devise, Inter Optode } \\
\text { Distance (IOD), position, wave } \\
\text { specifications }\end{array}$ & Physical task & $\begin{array}{l}\text { Type of task and } \\
\text { exercise intensity }\end{array}$ & Outcome \\
\hline $\begin{array}{l}\text { Shibuya et al. } \\
\text { (2004) }\end{array}$ & $\begin{array}{l}5 \mathrm{M} \\
\text { A: } 24.6 \pm 0.4 \text { year } \\
\text { Length: } \\
175.3 \pm 1.2 \mathrm{~cm} \\
\text { Mass: } \\
62.9 \pm 1.1 \mathrm{~kg} \\
\mathrm{VO}_{2 \text { peak: }} \\
48.4 \pm 1.3 \\
\mathrm{ml} / \mathrm{kg} / \mathrm{min} \\
\text { Training status: / }\end{array}$ & $\begin{array}{l}\text { IOD: } 50 \mathrm{~mm} \\
\text { Position: forehead } \\
\text { 780, 810, and } 830 \mathrm{~nm}\end{array}$ & $\begin{array}{l}\text { (Cycling) } \\
120 \% \mathrm{VO}_{2 \text { peak }} \text { exhaustive } \\
\text { exercise test }\end{array}$ & $\begin{array}{l}\text { TTE } \\
- \\
120 \% \text { VO2peak } \\
- \\
\text { Supramaximal }\end{array}$ & $\begin{array}{l}\text { - } \mathrm{SaO}_{2}=\text { throughout test } \\
\text { - } \mathrm{VO}_{2}, \mathrm{~V}_{\mathrm{E}} \text {, and } \mathrm{HR} \uparrow \text { gradually over time } \\
\text { - }\left[\mathrm{HbO}_{2}\right] \uparrow \text { first } 30 \mathrm{~s} \\
\text { - }\left[\mathrm{tHb} \text { and }\left[\mathrm{HbO} \mathrm{O}_{2}\right]=\text { throughout test }\right. \\
\text { - End of exercise: }[\mathrm{tH} \mathrm{Hb}] \text { and }\left[\mathrm{HbO}_{2}\right] \downarrow \text { from pre-exercise level }\end{array}$ \\
\hline $\begin{array}{l}\text { Suzuki et al. } \\
\text { (2004) }\end{array}$ & $\begin{array}{l}7 \mathrm{M}, 2 \mathrm{~F} \\
\text { A: } 28.1 \pm 7.4 \text { years } \\
\text { Training status: / }\end{array}$ & $\begin{array}{l}\text { OMM-2001, } \\
\text { Shimadzu, Kyoto, Japan } \\
\text { IOD: / } \\
\text { Position: anatomical 3-D T1 weighted } \\
\text { MRI scan was performed, marking the } \\
\text { optode location } \\
\text { 780, } 805 \text {, and } 830 \mathrm{~nm} \\
\text { Continuous wave }\end{array}$ & $\begin{array}{l}\text { (Running) } \\
3 \text { treadmill locomotor tasks of } \\
90 \mathrm{~s} \text {. each following } 30 \mathrm{~s} \text { rest: } \\
\text { 1. Walking at } 3 \mathrm{~km} / \mathrm{h} \\
\text { 2. Walking at } 5 \mathrm{~km} / \mathrm{h} \\
\text { 3. Running at } 9 \mathrm{~km} / \mathrm{h} \\
\text { Randomized order }\end{array}$ & $\begin{array}{l}\text { Adapting speed } \\
- \\
3 \mathrm{~km} / \mathrm{h} \\
5 \mathrm{~km} / \mathrm{h} \\
9 \mathrm{~km} / \mathrm{h} \\
- \\
\text { Light to moderate }\end{array}$ & $\begin{array}{l}\text { - } \mathrm{HbO}_{2} \text { and } \mathrm{tHb} \uparrow \text { bilaterally before starting the locomotor tasks } \\
\text { especially at } 9 \mathrm{~km} / \mathrm{h} \text { and peaked before the treadmill speed got } \\
\text { steady. } \\
\text { - After reaching constant speed: } \mathrm{HbO}_{2} \text { and } \mathrm{tHb} \downarrow \text { and tended to } \\
\text { return to the baseline or below baseline during performing the } \\
\text { locomotor tasks. } \\
\text { - After stopping locomotion: temporal drops in } \mathrm{HbO}_{2} \text { before } \\
\text { returning to the baseline } \\
\text { - } \uparrow \text { in } \mathrm{HbO}_{2} \text { levels greatest at } 9 \mathrm{~km} / \mathrm{h} \text { but are = between } 3 \text { and } \\
5 \mathrm{~km} / \mathrm{h} \text {. } \\
\text { - } \mathrm{PFC} \text {-activation = greater during running at } 9 \mathrm{~km} / \mathrm{h} \text { vs. walking } \\
\text { at } 3 \text { and } 5 \mathrm{~km} / \mathrm{h}\end{array}$ \\
\hline $\begin{array}{l}\text { Billaut et al. } \\
\text { (2010) }\end{array}$ & $\begin{array}{l}11 \mathrm{M} \\
\text { A: } 24.8 \pm 4.2 \\
\text { Length: } \\
174.2 \pm 5.3 \mathrm{~cm} \\
\text { Mass: } \\
66.8 \pm 3.1 \mathrm{~kg} \\
\text { Fat } \%: \\
10.3 \pm 3.5 \% \\
\text { Training status: PL5 }\end{array}$ & $\begin{array}{l}\text { Oxymon MKIII; Artinis Medical Systems } \\
\text { b.v., Zetten, the Netherlands } \\
\text { IOD: } 45 \mathrm{~mm} \\
\text { Position: Fp1 and F3, according to the } \\
\text { modified international EEG 10-20 } \\
\text { system } \\
763 \text { and } 855 \mathrm{~nm} \\
\text { Continuous wave }\end{array}$ & $\begin{array}{l}\text { (Running) } \\
\text { T1: Familiarization trial } \\
\text { T2: } 5 \mathrm{~km} \mathrm{TT} \\
\bullet 6 \text { min self-paced warm up } \\
\bullet 5 \mathrm{~km} T\left(4^{\circ} \text { incline }\right)\end{array}$ & $\begin{array}{l}\Pi \\
- \\
\text { Self-paced } \\
- \\
\text { Near to maximal }\end{array}$ & $\begin{array}{l}\text { - HR: } \uparrow \text { rapidly but remained nearly constant between } 1.5 \text { and } \\
4 \mathrm{~km} \text { and peaked in the last } 0.5 \mathrm{~km} \\
\text { - SaO2: fell between } 3 \text { and } 5 \mathrm{~km} \\
\text { - RPE } \uparrow \text { throughout the trial } \\
\text { - RBV and Cox } \uparrow \text { until } 2.5 \mathrm{~km}\left(\uparrow \Delta\left[\mathrm{HbO}_{2}\right], \uparrow \Delta[\mathrm{HHb}] \text {, and }\right. \\
\uparrow \triangle[\mathrm{tH} \mathrm{Hb}] \text { ), } \\
\mathrm{O}=\text { between } 2.5 \text { and } 4.5 \mathrm{~km}, \mathrm{f} \\
\text { o Deoxygenation in the last } 0.5 \mathrm{~km} \text {, while RBV remained stable }\end{array}$ \\
\hline $\begin{array}{l}\text { Fumoto et al. } \\
\text { (2010) }\end{array}$ & $\begin{array}{l}9 \mathrm{M}, 1 \mathrm{~F} \\
\mathrm{~A}: 32 \pm 2.2 \\
\text { Length: } \\
174.2 \pm 5.3 \mathrm{~cm} \\
\text { Mass : } 66.8 \pm 3.1 \\
\mathrm{~kg} \\
\text { Fat\%: } 10.3 \pm 3.5 \\
\text { Training status: PL2 }\end{array}$ & $\begin{array}{l}\text { OMM3000; Shimadzu Co., Kyoto, } \\
\text { Japan } \\
\text { IOD: } 30 \mathrm{~mm} \\
\text { Position: anatomical 3-D T1weighted } \\
\text { MRI scan was performed, marking the } \\
\text { optode location } \\
780,805 \text {, and } 830 \mathrm{~nm}\end{array}$ & $\begin{array}{l}\text { (Cycling) } \\
15 \text { min at } 60 \text { RPM and } \\
\text { pre-determined intensity of } \\
12-13 \text { RPE }\end{array}$ & $\begin{array}{l}\text { Constant load with } \\
\text { fixed duration } \\
- \\
12-13 \text { RPE } \\
- \\
\text { Moderate }\end{array}$ & $\begin{array}{l}\text { - Gradual } \uparrow \text { in } \mathrm{HbO}_{2} \text { and } \mathrm{tHb} \text {, reached a steady state at the end } \\
\text { of PE (mainly in ventral PFC regions) } \\
\text { - Gradual } \downarrow \text { in } \mathrm{HbO}_{2} \text { and tHb were observed after stopping } \\
\text { - Small } \downarrow \text { in } \mathrm{HHb} \text { or no change in both the ventral and dorsal PFO }\end{array}$ \\
\hline $\begin{array}{l}\text { Miyazawa et al. } \\
\text { (2013) }\end{array}$ & $\begin{array}{l}10 \mathrm{M} \\
\text { A: } 20.0 \pm 1 \text { year } \\
\text { Length: } 170 \pm 5 \\
\text { cm } \\
\text { Mass: } 64 \pm 9 \mathrm{~kg} \\
\text { Training status: / }\end{array}$ & $\begin{array}{l}\text { NIRO200, Hamamatsu Photonics, } \\
\text { Hamamatsu, Japan } \\
\text { IOD : } 40 \mathrm{~mm} \\
\text { Position: left forehead, } \pm 3 \mathrm{~cm} \text { from } \\
\text { midline, just above supra-orbital ridge } \\
780 \mathrm{~nm}\end{array}$ & $\begin{array}{l}\text { (Cycling) } \\
\text { - } 4 \text { min incremental warm-up } \\
\text { until } 60 \% \text { of } \mathrm{HR}_{\max } \\
\text { - } 11 \text {-min constant load cycling } \\
\text { o facial cooling from minute } \\
5-8\end{array}$ & $\begin{array}{l}\text { Constant load with } \\
\text { fixed duration } \\
- \\
60 \% \mathrm{HR}_{\max } \\
- \\
\text { Light }\end{array}$ & $\begin{array}{l}\text { - } \mathrm{SBFhead} \text { and } \mathrm{HbO}_{2} \uparrow \text { during exercise and temporally } \downarrow \text { with } \\
\text { facial cooling } \\
\text { - } \mathrm{HbO}_{2} \text { and tHb changes correlated with the relative changes in } \\
\mathrm{SBFhead} \\
\text { - } \mathrm{HHb} \text { and TOI did not change significantly with either exercise or } \\
\text { facial cooling } \\
\text { - Forehead TOI was not affected by exercise or facial cooling }\end{array}$ \\
\hline
\end{tabular}

(Running)

1: Familiarization tria

- 6 min self-paced warm up

- $5 \mathrm{~km}$ Tा (4ㅇincline)

Cycling

min at 60 RPM an

determined intensity of

$-$

Constant load with

fixed duration

$60 \% \mathrm{HR}_{\max }$

Light 
TABLE 5A | (Continued)

\begin{tabular}{|c|c|c|c|c|c|}
\hline Study & $\begin{array}{l}\text { Sample } \\
\text { characteristics } \\
\text { (Mean } \pm \text { SD) }\end{array}$ & $\begin{array}{l}\text { NIRS-devise, Inter Optode } \\
\text { Distance (IOD), position, wave } \\
\text { specifications }\end{array}$ & Physical task & $\begin{array}{l}\text { Type of task and } \\
\text { exercise intensity }\end{array}$ & Outcome \\
\hline $\begin{array}{l}\text { Rupp et al. } \\
\text { (2013) }\end{array}$ & $\begin{array}{l}10 \mathrm{M} \\
\text { A: } 37 \pm 7 \text { year } \\
\text { Length: } 180 \pm 5 \\
\text { cm } \\
\text { Mass: } 73 \pm 7 \mathrm{~kg} \\
\text { Training status: PL2 }\end{array}$ & $\begin{array}{l}\text { Oxymon III, Artinis, the Netherlands } \\
\text { IOD: } 35 \mathrm{~mm} \\
\text { Position Fp1 and F3, according to the } \\
\text { modified international EEG 10-20 } \\
\text { system } \\
780 \text { and } 850 \mathrm{~nm} \\
\text { Continuous wave }\end{array}$ & $\begin{array}{l}\text { (Cycling) } \\
4 \text {-h cycling exercise ( } 45 \% \text { max } \\
\text { aerobic power output) } \\
3 \text { consecutive } 80 \text {-min-bouts } \\
\text { (B1, B2, B3), separated by } \\
25 \text {-min of neuromuscular } \\
\text { function testing }\end{array}$ & $\begin{array}{l}\text { Constant load with } \\
\text { fixed duration } \\
- \\
45 \% \text { of maximal } \\
\text { aerobic power output } \\
- \\
\text { Vigorous }\end{array}$ & $\begin{array}{l}\text { - } \mathrm{MVC} \downarrow \text { after } \mathrm{B} 1, \mathrm{~B} 2 \text { and } \mathrm{B} 3(-11,-19 \text {, and }-25 \%) \\
\text { - } \mathrm{RPE} \uparrow \text { throughout the } 3 \text { bouts } \\
\text { - } \Delta\left[\mathrm{HbO}_{2}\right] \text { and } \Delta[\mathrm{tH} \mathrm{Hb} \uparrow \text { during } \mathrm{B} 1 \text { reaching a plateau after } \\
\sim 40 \mathrm{~min} \\
\text { - }[\mathrm{HHb} \text { progressively } \uparrow \text { during } \mathrm{B} 1 \text { from } 20 \text { to } 80 \text { min and showed } \\
\text { a main effect of bout throughout the protocol } \\
\text { - } \Delta[\mathrm{HH}] \uparrow \text { during } \mathrm{B} 2 \text { and } \mathrm{B} 3 \text { sign. reduced compared to } \mathrm{B} 1\end{array}$ \\
\hline $\begin{array}{l}\text { Giles et al. } \\
\text { (2014) }\end{array}$ & $\begin{array}{l}14 \mathrm{M} ; 10 \mathrm{~F} \\
\mathrm{~A}: 20.21 \pm 2.38 \\
\text { year } \\
\text { BMl: } 22.51 \pm 2.72 \\
\text { Training status: PL2 }\end{array}$ & $\begin{array}{l}\text { fNIR Imager } 1100 \text {, fNIR Devices LLC, } \\
\text { Potomac, Maryland, United States } \\
\text { IOD: } 25 \mathrm{~mm} \\
\text { Position: forehead; bottom of the probe } \\
\text { at the Fpz, (international 10-20 system) } \\
730 \text { and } 850 \mathrm{~nm} \\
\text { Continuous wave }\end{array}$ & $\begin{array}{l}\text { (Cycling) } \\
\text { 30-min recumbent cycling } \\
\text { followed by a } 5 \text {-min cool down } \\
\left.\text { T1: low load ( } 52 \% H R_{\max }\right) \\
\text { T2: moderate load }\left(68 \% H R_{\max }\right) \\
\text { T3: high load }\left(84 \% H R_{\max }\right) \\
\text { Counterbalanced }\end{array}$ & $\begin{array}{l}\text { Constant load with } \\
\text { fixed duration } \\
- \\
52 \% H R_{\max } \\
68 \% \mathrm{HR}_{\max } \\
84 \% \mathrm{HR}_{\max } \\
- \\
\text { Light } \\
\text { Moderate } \\
\text { vigorous }\end{array}$ & 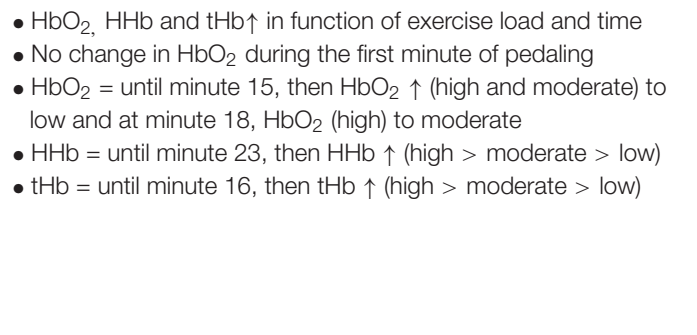 \\
\hline $\begin{array}{l}\text { Santos- } \\
\text { Concejero et al. } \\
\text { (2015) }\end{array}$ & $\begin{array}{l}10 \mathrm{M} \\
\text { A: } 23.7 \pm 4.2 \text { year } \\
\text { Length: } \\
170.5 \pm 6.3 \mathrm{~cm} \\
\text { Mass: } \\
54.8 \pm 6.3 \mathrm{~kg} \\
\text { BMl: } 18.8 \pm 1.3 \\
\text { Fat } \%: 8.7 \pm 0.5 \% \\
\text { Training status: PL5 }\end{array}$ & $\begin{array}{l}\text { NIRO-200X, Hamamatsu, Japan } \\
\text { IOD: } 30 \mathrm{~mm} \\
\text { Position: left prefrontal lobe between } \\
\text { Fp1 and F3, according to the modified } \\
\text { international EEG } 10-20 \text { system. } \\
735,810 \text {, and } 850 \mathrm{~nm} \\
\text { Continuous wave }\end{array}$ & $\begin{array}{l}\text { (Running) } \\
\text { T1: 5-km time trial } \\
\text { T2: MIT } \\
\text { T3: constant speed running } \\
\text { bouts (running economy } \\
\text { determination) }\end{array}$ & $\begin{array}{l}\Pi \\
- \\
\text { Self-selected speed } \\
- \\
\text { Near to maximal }\end{array}$ & 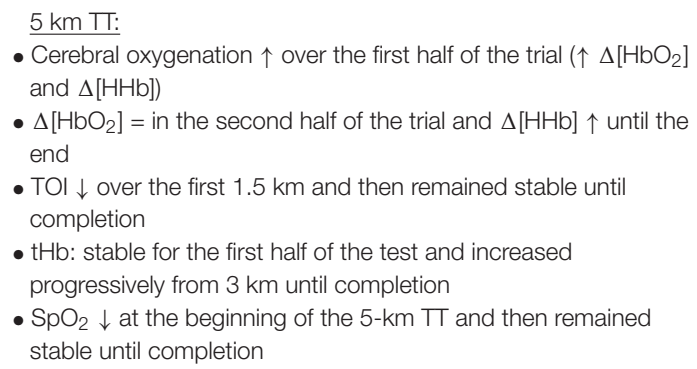 \\
\hline $\begin{array}{l}\text { Pires et al. } \\
\text { (2016) }\end{array}$ & $\begin{array}{l}10 \mathrm{M} \\
\text { A: } 32.9 \pm 7.3 \text { year } \\
\text { Length: } \\
175.7 \pm 5.9 \mathrm{~cm} \\
\text { Mass: } 75.9 \pm 9.0 \\
\mathrm{~kg} \\
\text { Fat\%: } 10.5 \pm 5.2 \% \\
\text { Training status: PL3 }\end{array}$ & $\begin{array}{l}\text { CW6- TechEn, Milford, MA, } \\
\text { United States } \\
\text { IOD: } 45 \mathrm{~mm} \\
\text { Position: prefrontal lobe at the Fp1 } \\
\text { position (international 10-20 system) } \\
690 \text { and } 830 \mathrm{~nm} \\
\text { Continuous wave }\end{array}$ & $\begin{array}{l}\text { (Cycling) } \\
\text { T1: Familiarization } \\
\text { T2: MIT } \\
\text { T3: Self-paced } 4 \text { km cycling TT }\end{array}$ & $\begin{array}{l}\Pi \\
- \\
\text { Self-selected power } \\
- \\
\text { Near to maximal }\end{array}$ & $\begin{array}{l}\text { - } \Delta\left[\mathrm{HbO}_{2}\right], \Delta[\mathrm{HHb}] \text {, and } \Delta[\mathrm{tHb}] \uparrow \text { up to } \sim 70 \% \text { of both MIT and } \\
\prod 4 \mathrm{~km} \text {, and } \downarrow \text { afterward } \\
\text { - } \Delta\left[\mathrm{HbO}_{2}\right] \text { was lower in } \Pi 4 \mathrm{~km} \text { than MIT at 20, 30, 40, 50, and } \\
60 \% \text {, but higher at } 100 \% \text { of the exercise duration } \\
\text { - Greater RPE slope in MIT than in } T 4 \mathrm{~km}\end{array}$ \\
\hline
\end{tabular}




\begin{tabular}{|c|c|c|c|c|c|}
\hline Study & $\begin{array}{l}\text { Sample } \\
\text { characteristics } \\
\text { (Mean } \pm \text { SD) }\end{array}$ & $\begin{array}{l}\text { NIRS-devise, Inter Optode } \\
\text { Distance (IOD), position, wave } \\
\text { specifications }\end{array}$ & Physical task & $\begin{array}{l}\text { Type of task and } \\
\text { exercise intensity }\end{array}$ & Outcome \\
\hline $\begin{array}{l}\text { Santos- } \\
\text { Concejero et al. } \\
\text { (2017) }\end{array}$ & $\begin{array}{l}15 \mathrm{M} \\
\text { A: } 23.7 \pm 4.2 \text { year } \\
\text { Length: } \\
170.5 \pm 6.3 \mathrm{~cm} \\
\text { Mass: } \\
54.8 \pm 6.3 \mathrm{~kg} \\
\text { Training status: PL5 }\end{array}$ & $\begin{array}{l}\text { NIRO-200X, Hamamatsu, Japan } \\
\text { IOD: } 30 \mathrm{~mm} \\
\text { Position: left prefrontal lobe between } \\
\text { Fp1 and F3, according to the modified } \\
\text { international EEG } 10-20 \text { system. } \\
\text { 735, } 810 \text {, and } 850 \mathrm{~nm} \\
\text { Continuous wave }\end{array}$ & $\begin{array}{l}\text { (Running) } \\
\text { T1: 5-km time trial, } \\
\text { T2: MIT } \\
\text { T3: Fatigue training test (FTT) }\end{array}$ & $\begin{array}{l}\text { T1: Tा } \\
\text { Self-selected speed } \\
- \\
\text { T2: MIT } \\
- \\
\text { T3: FTा } \\
\text { 5\% faster than } 5 \mathrm{~km} \mathrm{TT} \\
\text { pace } \\
- \\
\text { Near to maximal }\end{array}$ & 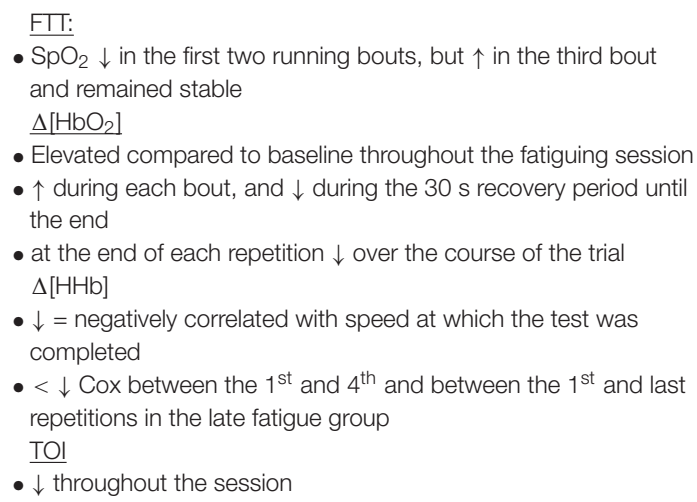 \\
\hline $\begin{array}{l}\text { Ohyanagi et al. } \\
\text { (2018) }\end{array}$ & $\begin{array}{l}6 \mathrm{M}, 6 \mathrm{~F} \\
\text { A: } 20.0 \text { year } \\
\text { Training status: / }\end{array}$ & $\begin{array}{l}\text { OMM3000; Shimadzu Co., Kyoto, } \\
\text { Japan } \\
\text { IOD: } 30 \mathrm{~mm} \\
\text { Position: Fpz position of the } \\
\text { international } 10-20 \text { system } \\
\text { 780, } 805 \text {, and } 830 \mathrm{~nm} \\
\text { Continuous wave }\end{array}$ & $\begin{array}{l}\text { (Cycling) } \\
\text { T1: MIT } \\
\text { T2: Experimental trial (randomly } \\
\text { assigned to upright or supine } \\
\text { position): } \\
\text { - 4-min pre exercise rest } \\
\text { - 4-min warm-up } \\
\text { - 20-min at } 50 \% \mathrm{VO}_{2} \text { max } \\
\text { - 15-min PER }\end{array}$ & $\begin{array}{l}\text { Constant load with } \\
\text { fixed duration } \\
- \\
50 \% \mathrm{VO}_{2} \max \\
- \\
\text { Moderate }\end{array}$ & $\begin{array}{l}\mathrm{HbO}_{2:} \\
\text { - } \uparrow \text { from } 3 \text { to } 6 \text { min during the } 20 \text {-min main exercise } \\
\text { - Steady from min } 6 \text { until the end of exercise } \\
\text { SBF: } \\
\text { - } \uparrow \text { gradually throughout the exercise } \\
\text { - MAP } \uparrow \text { during the first } 3 \text { min of exercise and then } \downarrow \text { slowly } \\
\text { throughout the exercise phase }\end{array}$ \\
\hline $\begin{array}{l}\text { Radel et al. } \\
(2017)\end{array}$ & $\begin{array}{l}15 \mathrm{M}, 7 \mathrm{~F} \\
\text { A: } 21.3 \pm 2.1 \text { year } \\
\text { Length: } \\
174.7 \pm 6.4 \mathrm{~cm} \\
\text { Mass: } 67.0 \pm 10.6 \\
\mathrm{~kg} \\
\text { Peak HR: } \\
186.5 \pm 10.9 \\
\text { PAP: } 286.1 \pm 79.3 \\
\text { Training status: / }\end{array}$ & $\begin{array}{l}\text { Oxymon Mk II, Artinis Medical Systems, } \\
\text { Zetten, the Netherlands } \\
\text { IOD: } 40 \mathrm{~mm} \\
\text { Position: AF2h and F6h sites of the } \\
\text { extended } 10-5 \mathrm{EEG} \text { system } \\
764 \text { and } 858 \mathrm{~nm} \\
\text { Continuous wave }\end{array}$ & $\begin{array}{l}\text { (Cycling) } \\
\text { T1: MIT } \\
\text { T2 and T3: } \\
60 \% \text { PAP for 10-min } \\
\text { Participants expected one trial } \\
\text { to last } 60 \text {-min }\end{array}$ & $\begin{array}{l}\text { Constant load with } \\
\text { fixed duration } \\
- \\
60 \% \text { PAP } \\
- \\
\text { Near to maximal }\end{array}$ & $\begin{array}{l}\text { - lower general }\left[\mathrm{HbO}_{2}\right] \text { in the first period vs. second- and third } \\
\text { periods of time } \\
\text { - rdlPFC: }\left[\mathrm{HbO}_{2}\right] \text { less elevated in the 60-min than in the 10-min } \\
\text { condition } \\
\text { - rmPFC: }\left[\mathrm{HbO}_{2}\right] \text { higher in the } 60 \text {-min than in the 10-min } \\
\text { condition } \\
\text { - Attention was less focused on the exercise trial in the 60-min } \\
\text { than in the 10-min condition } \\
\text { - RPE = }\end{array}$ \\
\hline $\begin{array}{l}\text { Tsubaki et al. } \\
\text { (2018) }\end{array}$ & $\begin{array}{l}8 \mathrm{M}, 4 \mathrm{~F} \\
\text { A: } 21.3 \pm 0.7 \text { year } \\
\text { Training status: / }\end{array}$ & $\begin{array}{l}\text { OMM3000; Shimadzu Co., Kyoto, } \\
\text { Japan } \\
\text { IOD: } 30 \mathrm{~mm} \\
\text { Position: Cz position of the International } \\
\text { 10-20 System } \\
\text { 780, } 805 \text {, and } 830 \mathrm{~nm} \\
\text { Continuous wave }\end{array}$ & $\begin{array}{l}\text { (Cycling) } \\
\text { T1: MIT } \\
\text { T2 : Experimental trial } \\
\text { - 3-min rest } \\
\text { - 20-min at to 50\% VO } 2 \text { peak } \\
\text { - 15-min PER }\end{array}$ & $\begin{array}{l}\text { Constant load with } \\
\text { fixed duration } \\
- \\
50 \% \mathrm{VO}_{2 \text { peak }} \\
- \\
\text { Moderate }\end{array}$ & $\begin{array}{l}\text { - } \mathrm{HbO}_{2} \uparrow \text { between start and the last } 5 \text { min of the exercise } \\
\text { (R-PFC) } \\
\text { - } \mathrm{HbO} \text { did not return to pre-exercise levels during the } 15 \text {-min } \\
\text { PER } \\
\text { - } \mathrm{HbO} \text {. higher during the last } 5 \text { min of PER than during the } \\
\text { pre-exercise rest period } \\
\text { - } \mathrm{HbO}_{2}=\text { between the last } 5 \text { min of exercise and } 15 \text {-min PER. } \\
\text { - } \mathrm{SBF} \text { and MAP } \uparrow \text { during exercise and } \downarrow \text { during PER } \\
\text { - SBF and MAP = between pre-exercise rest phase and the last } \\
5 \text { min of the } 15 \text {-min PER. }\end{array}$ \\
\hline
\end{tabular}

780,805 , and $830 \mathrm{~nm}$

Oxymon Mk II, Artinis Medical Systems, Zetten, the Netherland

system

Mass: $67.0 \pm 10.6$

$\mathrm{kg}$

$186.5 \pm 10.9$ 
TABLE 5A | (Continued)

\begin{tabular}{|c|c|c|c|c|c|}
\hline Study & $\begin{array}{l}\text { Sample } \\
\text { characteristics } \\
\text { (Mean } \pm \text { SD) }\end{array}$ & $\begin{array}{l}\text { NIRS-devise, Inter Optode } \\
\text { Distance (IOD), position, wave } \\
\text { specifications }\end{array}$ & Physical task & $\begin{array}{l}\text { Type of task and } \\
\text { exercise intensity }\end{array}$ & Outcome \\
\hline $\begin{array}{l}\text { Hiura et al. } \\
\text { (2018) }\end{array}$ & $\begin{array}{l}\text { PET-study } \\
12 \mathrm{M} \\
\text { A: } 21.1 \pm 2 \text { year } \\
\text { Training status: / } \\
\text { NIRS-study } \\
12 \mathrm{M} \\
\text { A: } 22.5 \pm 2.9 \text { year } \\
\text { Training status: / }\end{array}$ & $\begin{array}{l}\text { NIRS : } \\
\text { Spectratech, OEG-16, Yokohama, } \\
\text { Japan } \\
\text { IOD:30 mm } \\
\text { Position: Fpz position of the } \\
\text { international 10-20 system } \\
770 \text { and } 840 \mathrm{~nm} \\
\text { PET: } \\
\text { Discovery PET/ CT } 710 \text { scanner (GE } \\
\text { Healthcare, Milwaukee, WI }\end{array}$ & $\begin{array}{l}\text { (Cycling) } \\
15 \text {-min at 30\% HR reserve }\end{array}$ & $\begin{array}{l}\text { Constant load with } \\
\text { fixed duration } \\
- \\
\text { 30\% HR reserve } \\
- \\
\text { Light }\end{array}$ & $\begin{array}{l}\text { - } \Delta \mathrm{HbO}_{2} \uparrow \text { at Ex2 but did not change at Ex1 } \\
\text { - } \Delta \mathrm{HHb}=\text { during exercise } \\
\text { - } \mathrm{rCBF} \uparrow \text { at Ex } 1 \text { but }=\text { at Ex2. } \\
\text { - } \mathrm{PET} \mathrm{CO} 2 \uparrow \text { at Ex1 and }=\text { at Ex } 2 . \\
\text { - } \mathrm{MBP} \uparrow \text { during exercise but } \downarrow \text { at Ex2 compared with Ex1 }\end{array}$ \\
\hline $\begin{array}{l}\text { Kounalakis and } \\
\text { Geladas (2012) }\end{array}$ & $\begin{array}{l}12 \mathrm{M} \\
\text { A: } 23.4 \pm 3.8 \text { year } \\
\text { Length: } \\
179 \pm 7 \mathrm{~cm} \\
\text { Mass: } \\
78.3 \pm 6.7 \mathrm{~kg} \\
\text { Fat } \%: 10.4 \pm 3.6 \% \\
\text { Training status: / }\end{array}$ & $\begin{array}{l}\text { NIRS, In Spectra325, Hutchinson } \\
\text { Technology Inc., Hutchinson, Minn } \\
\text { IOD: / } \\
\text { Position: middle of the forehead, just } \\
\text { below the hairline } \\
720 \text { and } 760 \mathrm{~nm}\end{array}$ & $\begin{array}{l}\text { (Cycling) } \\
2 \text { preliminary MIT at } 40 \text { - and } 80 \\
\text { RPM } \\
2 \text { experimental trials: } \\
\text { T1: } 40 \text { RPM } \\
\text { T2: } 80 \text { RPM } \\
\text { - } 8 \text { min warm-up ( } 50 \% \\
\text { VO }{ }_{2 \text { peak }} \text { ) } \\
\text { - Three } 5 \text { s MVC } \text { isometric (knee) } \\
\text { - } 25 \text { min rest on the cycle } \\
\text { ergometer } \\
\text { - } 90 \text { min at } 58-60 \% \mathrm{VO}_{2 \text { peak }}\end{array}$ & $\begin{array}{l}\text { Constant load with } \\
\text { fixed duration } \\
- \\
58-60 \% \mathrm{VO}_{2 p e a k} \\
- \\
\text { Moderate }\end{array}$ & $\begin{array}{l}\text { - } \mathrm{O}_{2} \text {-cost during } 5 \text { min of unloaded pedaling was significantly } \\
\text { higher at } 80 \text { - than at } 40 \mathrm{RPM} \\
\text { - First } 8 \text { min: } \mathrm{VO}_{2} \text { sign. } \uparrow(\sim 250 \mathrm{ml}) \text { at } 80 \text { than at } 40 \mathrm{RPM} \text {. } \\
\text { - First } 8 \text { min: } \mathrm{HR}= \\
\text { Prefrontal oxygenation: } \\
\text { - } \Delta\left[\mathrm{HbO}_{2}\right]: \uparrow \text { from start to end of exercise } \\
\text { - } \Delta[\mathrm{tHb}] \& \Delta\left[\mathrm{HbO}_{2}\right] \text { : lower at } 80 \text { vs. } 40 \text { RPM at the end of } \\
\text { exercise } \\
\text { - } \Delta[\mathrm{HH} \mathrm{Hb}]: \text { lower at the end of exercise at } 80 \mathrm{RPM} \\
\text { - } \Delta[\mathrm{HH} \mathrm{Hb}]=\text { throughout exercise at } 40 \mathrm{RPM} \\
\text { - } \mathrm{SpO}_{2}: \downarrow \text { at } 80 \text { than at } 40 \mathrm{RPM} \text { during exercise }\end{array}$ \\
\hline $\begin{array}{l}\text { Takehara et al. } \\
\text { (2017) }\end{array}$ & $\begin{array}{l}5 \mathrm{M}, 8 \mathrm{~F} \\
\text { A: } 21.2 \pm 0.6 \text { year } \\
\text { Length: } \\
174.7 \pm 6.4 \mathrm{~cm} \\
\text { Mass: } 67.0 \pm 10.6 \\
\mathrm{~kg} \\
\text { Peak HR: } \\
186.5 \pm 10.9 \\
\text { PAP: } 286.1 \pm 79.3 \\
\text { Training status: / }\end{array}$ & $\begin{array}{l}\text { OMM3000, Shimadzu Co., Kyoto, } \\
\text { Japan } \\
\text { IOD: } 30 \mathrm{~mm} \\
\text { Position: Cz position of the International } \\
\text { 10-20 System }\end{array}$ & $\begin{array}{l}2 \text { trials: } \\
\text { Trial 1: } 30 \% \mathrm{VO}_{2 \text { peak }} \\
\text { Trial 2: } 50 \% \mathrm{VO}_{2 \text { peak }} \\
\text { - } 180 \text { s rest period, } \\
\quad 10 \text { min continuous } \\
\text { cycling exercise at } 55 \mathrm{RPM}\end{array}$ & $\begin{array}{l}\text { Constant load with } \\
\text { fixed duration } \\
- \\
30 \% \mathrm{VO}_{2 \text { peak }} \\
50 \% \mathrm{VO}_{2 \text { peak }} \\
- \\
\text { Light } \\
\text { Moderate }\end{array}$ & 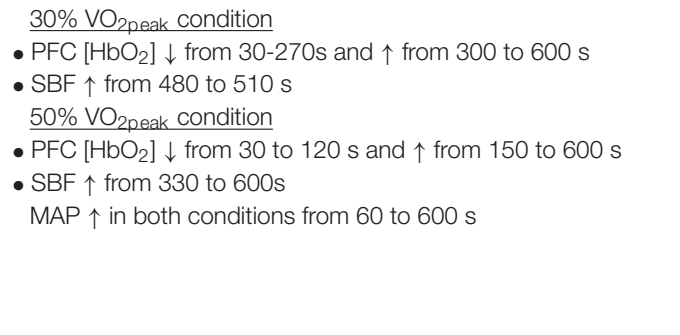 \\
\hline Ide et al. (1999) & $\begin{array}{l}7 \mathrm{M}, 7 \mathrm{~F} \\
\text { A: } 20.6 \pm 1.4 \text { year } \\
\text { Length: } \\
171.0 \pm 7.5 \mathrm{~cm} \\
\text { Mass: } \\
69.3 \pm 7.3 \mathrm{~kg} \\
\text { BMl: } 23.4 \pm 2.0 \\
\text { Training status: / }\end{array}$ & $\begin{array}{l}\text { NIRO 500, Hamamatsu Photonics, } \\
\text { Hamamatsu, Japan. } \\
\text { IOD: } 45 \mathrm{~mm} \text { apart, } \\
\text { Position: forehead } \\
775,825 \text {, and } 850 \mathrm{~nm}\end{array}$ & $\begin{array}{l}\text { T1: MIT } \\
\text { T2: experimental trial } \\
\text { - } 10 \text { min rest } \\
\text { - } 10 \text { min cycling at } 30 \% \\
\mathrm{VO}_{2} \text { max } \\
\text { - } 10 \text { min cycling at } 60 \% \\
\mathrm{VO}_{2} \text { max } \\
\text { - } 10 \text { min PER }\end{array}$ & $\begin{array}{l}\text { Constant load with } \\
\text { fixed duration } \\
- \\
30 \% \mathrm{VO}_{2} \max \\
60 \% \mathrm{VO}_{2} \max \\
- \\
\text { Light } \\
\text { Moderate }\end{array}$ & $\begin{array}{l}\text { - } \Delta\left[\mathrm{HbO}_{2}\right]: \uparrow \text { in proportion to work rate and reached a maximal } \\
\text { level during the first few minutes of exercise } \\
\text { - } \mathrm{HHb} \text { and } t \mathrm{Hb}: \uparrow \\
\text { - MCA } \vee_{\text {mean: }} \uparrow \\
\text { - MAP and } \mathrm{HR}: \uparrow \text { with exercise intensity }\end{array}$ \\
\hline
\end{tabular}

M, man; F, female; A, age; Y, Years; BMI, Body Mass Index; Fat\%, body fat; KG, kilogram; Cm, Centimeter; mm, Millimeter; VO2peak, VO 2 max Maximal Oxygen Uptake; PL, Performance Level; NIRS, Near Infrared Spectroscopy; IOD, Inter Optode Distance; TTE, Time To Exhaustion; $\mathrm{SaO}_{2}$, Arterial Oxygen Saturation; $\mathrm{VO}_{2}$, Oxygen Uptake; $\mathrm{V}_{\mathrm{E}}$, Ventilation; HR, Heart Rate; tHb, Total Hemoglobin; HbO $\mathrm{H}_{2}$, Oxygenated hemoglobin; HHb, Deoxygenated Hemoglobin; MRI, Magnetic Resonance Imaging; Sec, Second; Min, Minute; Km, Kilometer; H, Hour; BP, Blood Pressure; PFC, Prefrontal Cortex; PMC, Premotor Cortex; m-SMC, Medial SensoriMotor Cortex; TT, Time-Trial; EEG, Electroencephalography; RPE, Rating of Perceived Exertion; CPT(RM), Constant-Power Test (Respiratory Maneuver); RPM, Rotations per Minute; SBF, Skin Blood Flow; TOI, Tissue Oxygenation Index; MVC, Maximal Voluntary Contraction; MIT, Maximal Incremental Exercise Test; Cox, Cerebral Oxygenation; FIT, Fatigue Training Test; PER, Post-Exercise Rest; MAP, Mean Arterial Pressure; PAP, Peak Aerobic Power; T, Trial. 
TABLE 5B | Overview of the results within "maximal incremental exercise (MIT)".

\begin{tabular}{|c|c|c|c|c|c|}
\hline Study & $\begin{array}{l}\text { Sample } \\
\text { characteristics } \\
\text { (Mean } \pm \text { SD) }\end{array}$ & $\begin{array}{l}\text { NIRS-devise, Inter Optode } \\
\text { Distance (IOD), position, wave } \\
\text { specifications }\end{array}$ & Physical task & $\begin{array}{l}\text { Type of task and } \\
\text { exercise intensity }\end{array}$ & Outcome \\
\hline $\begin{array}{l}\text { Bhambhani } \\
\text { et al. (2007) }\end{array}$ & $\begin{array}{l}7 \mathrm{M} \\
\text { A: } 26.7 \pm 8.6 \text { year } \\
\text { Length: } 178 \pm 0.6 \\
\text { cm } \\
\text { Mass : }: 77.5 \pm 9.3 \\
\mathrm{~kg} \\
\text { BMl: } 24.1 \pm 2.5 \\
\text { Training status: PL1 }\end{array}$ & $\begin{array}{l}\text { MicroRunman, NIM Inc., Philadelphia, } \\
\text { PA } \\
\text { IOD: } 40 \mathrm{~mm} \\
\text { Position: left pre-frontal lobe, } \pm 3 \mathrm{~cm} \\
\text { from midline, just above the } \\
\text { supra-orbital ridge }\end{array}$ & $\begin{array}{l}\text { (Cycling) } \\
\text { - } 2 \text {-min rest + baseline } \\
\text { measurement } \\
\text { - MIT: } 30 \text { W/2-min until } \\
\text { exhaustion }\end{array}$ & $\begin{array}{l}\text { MIT } \\
- \\
\uparrow 30 \text { W/2-min }\end{array}$ & $\begin{array}{l}\text { - } \mathrm{PET}_{\text {co2 }} \uparrow \text { systematically until RCT-GEX } \\
\text { - After RCT-GEX: PET } \mathrm{CO2} \downarrow \text { continuously until exhaustion } \\
\text { - CBV and Cox } \uparrow \text { systematically during MIT, slightly beyond the } \\
\text { RCT-GEX-intensity, then continuous } \downarrow \text { until exhaustion } \\
\text { - } \uparrow \text { in Cbv }>\uparrow \text { in Cox } \\
\text { - Sign. difference RCT-GEX, } 76.9 \% \text { and RCT-NIRS, } 81.0 \% \text {, as } \\
\%^{2} \mathrm{VO}_{2 \max }\end{array}$ \\
\hline $\begin{array}{l}\text { Rupp and } \\
\text { Perrey (2008) }\end{array}$ & $\begin{array}{l}13 \mathrm{M} \\
\text { A: } 24.9 \pm 1.5 \text { year } \\
\text { Length: } \\
179.3 \pm 1.8 \mathrm{~cm} \\
\text { Mass: } \\
71.1 \pm 1.2 \mathrm{~kg} \\
\text { Training status: PL5 }\end{array}$ & $\begin{array}{l}\text { NIRO-300, Hamamatsu Photonics, } \\
\text { Japan } \\
\text { IOD: } 50 \mathrm{~mm} \\
\text { Position: Fp1 and F3, according to the } \\
\text { modified international EEG 10-20 } \\
\text { system } \\
775,810,850 \text {, and } 905 \mathrm{~nm}\end{array}$ & $\begin{array}{l}\text { (Cycling) } \\
\text { 2-min rest + baseline } \\
\text { measurement } \\
\text { - } 3 \text {-min warm-up at } 60 \mathrm{~W} \\
\text { MIT: } 30 \mathrm{~W} / \text { min until exhaustion } \\
\text { Pre- and post-MIT: } 2 \text { MVICs } \\
\text { (knee extensor) }\end{array}$ & $\begin{array}{l}\text { MIT } \\
- \\
60+30 \mathrm{~W} / \mathrm{min}\end{array}$ & $\begin{array}{l}\text { - } \frac{P F C-o x y g e n a t i o n}{} \Delta[\mathrm{tH} b] \uparrow \text { until } \mathrm{VT} 2 \text { and then stabilized } \\
\text { - } \Delta[\mathrm{HH} \mathrm{H}] \uparrow \text { with the workload after warm-up until exhaustion and } \\
\text { finally } \downarrow \text { toward resting values during recovery } \\
\text { - } \Delta[\mathrm{HbO2}] \uparrow \text { from warm-up to } V T 2 \text {, then dropped until } \\
\text { exhaustion and finally } \uparrow \text { over resting values during recovery } \\
\text { - Exhaustion and finally overshot the resting values during } \\
\text { recovery }\end{array}$ \\
\hline $\begin{array}{l}\text { Timinkul et al. } \\
\text { (2008) }\end{array}$ & $\begin{array}{l}10 \mathrm{M} \\
\text { A: } 21.4 \pm 0.6 \text { year } \\
\text { Length: } 175 \pm 1.6 \\
\text { cm } \\
\text { Mass: } 68 \pm 3.6 \mathrm{~kg} \\
\text { Training status: PL1 }\end{array}$ & $\begin{array}{l}\text { NIRO-300, Hamamatsu Photonics, } \\
\text { Japan } \\
\text { IOD: } 50 \mathrm{~mm} \\
\text { Position: Fp1 and F3, according to the } \\
\text { modified international EEG 10-20 } \\
\text { system } \\
775,810,850 \text {, and } 905 \mathrm{~nm} \\
\text { Continuous wave }\end{array}$ & $\begin{array}{l}\text { (Cycling) } \\
\text { - } 2 \text {-min rest + baseline } \\
\text { measurement } \\
\text { - MIT: } 0+25 \mathrm{~W} / 3 \text { min until } 150 \\
\mathrm{~W}, \\
150+25 \mathrm{~W} / \text { min until } \\
\text { exhaustion }\end{array}$ & $\begin{array}{l}\text { MIT } \\
- \\
150+25 \mathrm{~W} / \mathrm{min}\end{array}$ & $\begin{array}{l}\text { - HR and VO2 } \uparrow \text { progressively } \\
\text { - Correlation between [Bla] and } \mathrm{HHb} \text { and } \mathrm{HR} \text { and } \mathrm{HbO} 2 \\
\text { - TOI response showed a small } \uparrow(2-7 \% \text { ) or no change (one } \\
\text { subject) with } \uparrow \text { workload. However, before all-out, it began to } \downarrow \\
\text { gradually } \\
\text { - } \mathrm{PETCO} \uparrow \uparrow \text { gradually with } \uparrow \text { workload and reached its peak, } \\
\text { then } \downarrow \text { gradually until all-out } \\
3 \text { distinct phases } \\
\text { 1. Linear oxygenation phase (until blood volume threshold) } \\
\text { - } \mathrm{HbO}_{2} \text { : gradual } \uparrow \text { in } \mathrm{VO}_{2} \text { from start till } 15 \text { min (100 W). } \\
\text { - } \mathrm{HHb} \text { : small } \downarrow \\
\text { 2. Hyper-oxygenation phase: } \\
\text { - } \mathrm{HbO}_{2}: \text { rapid cerebral oxygen intake after } 15 \text {-min (100 W) } \\
\text { - } \mathrm{HHb}: \pm= \\
\text { 3. Desaturation phase: } \\
\text { - } \mathrm{HbO} \text { : oxygenation continuously } \downarrow \text { from respiratory } \\
\text { compensation threshold until exhaustion } \\
\mathrm{HHb} \uparrow\end{array}$ \\
\hline $\begin{array}{l}\text { Tempest et al. } \\
\text { (2014) }\end{array}$ & $\begin{array}{l}13 \mathrm{M}, 12 \mathrm{~F} \\
\text { A: } 25.6 \pm 3.4 \text { year } \\
\text { Length: } \\
174.8 \pm 6.8 \mathrm{~cm} \\
\text { Mass: } \\
71.8 \pm 8.3 \mathrm{~kg} \\
\mathrm{BMI}: 23.5 \pm 2.2 \\
\mathrm{VO}_{2 \text { peak: }} \\
41.8 \pm 5.2 \mathrm{ml} / \mathrm{kg}^{-1 *} \mathrm{n} \\
\text { Training status: } \mathrm{PL} 1\end{array}$ & $\begin{array}{l}\text { Oxymon Mk II, Artinis Medical Systems, } \\
\text { Zetten, the Netherlands } \\
\text { IOD: } 40 \mathrm{~mm} \\
\text { Position: AF2 } \mathrm{h} \text { and F6 } \mathrm{h} \text { sites of the } \\
\text { extended } 10-5 \mathrm{EEG} \text { system } \\
\text { Continuous wave }\end{array}$ & $\begin{array}{l}\text { (Cycling) } \\
\text { - Recumbent cycle ergometer } \\
\text { MIT: } 20 \text { W/min until exhaustion }\end{array}$ & $\begin{array}{l}\text { MIT } \\
- \\
20 \mathrm{~W} / \mathrm{min}\end{array}$ & $\begin{array}{l}\text { - } \Delta \mathrm{HbO}_{2} \uparrow \text { from below } \mathrm{VT} \text { to } \mathrm{VT} \text { and } \mathrm{VT} \text { to } \mathrm{RCP} \text {, but remained } \\
\text { stable from RCP to End } \\
\text { - } \Delta \mathrm{HH} \text { bremained stable from below } \mathrm{VT} \text { to } \mathrm{VT} \text {, then } \uparrow \text { from } \mathrm{VT} \text { to } \\
\text { RCP and RCP to End } \\
\text { - } \Delta \text { tHb increased from below } \mathrm{VT} \text {, to } \mathrm{VT}, \mathrm{RCP} \text {, and End }\end{array}$ \\
\hline
\end{tabular}

A: $21.4 \pm 0.6$ year

Mass: $68 \pm 3.6 \mathrm{~kg}$ modified international EEG 10-20

-2-min rest + baseline

measurement

IOD: $40 \mathrm{~mm}$

exhaustion

measurement

MIT: $30 \mathrm{~W} / \mathrm{min}$ until exhaustion

Pre- and post-MIT: 2 MVICs

knee extensor)

2-min rest + baseline

measurement

W

$150+25 \mathrm{~W} / \mathrm{min}$ unt

xhaustion 
TABLE 5B | (Continued)

\begin{tabular}{|c|c|c|c|c|c|}
\hline Study & $\begin{array}{l}\text { Sample } \\
\text { characteristics } \\
\text { (Mean } \pm \text { SD) }\end{array}$ & $\begin{array}{l}\text { NIRS-devise, Inter Optode } \\
\text { Distance (IOD), position, wave } \\
\text { specifications }\end{array}$ & Physical task & $\begin{array}{l}\text { Type of task and } \\
\text { exercise intensity }\end{array}$ & Outcome \\
\hline $\begin{array}{l}\text { Jung et al. } \\
\text { (2015) }\end{array}$ & $\begin{array}{l}9 \mathrm{M} \\
\text { A: } 23-24 \text { year } \\
\text { Length: } 182 \pm 5 \\
\text { cm } \\
\text { Mass: } \\
77.6 \pm 4.6 \mathrm{~kg} \\
\text { Fat } \%: 11.0 \pm 2.8 \% \\
\text { Training status: PL3 }\end{array}$ & $\begin{array}{l}\text { Oxymon, Artinis, Zetten, The } \\
\text { Netherlands } \\
\text { IOD: } 25 \mathrm{~mm} \\
\text { Position: left PFC (Brodmann area 10, } \\
\text { MNI coordinates (x/y/z) -40, 50, 0) } \\
858 \text { and } 764 \mathrm{~nm} \\
\text { Continuous wave }\end{array}$ & $\begin{array}{l}\text { (Cycling) } \\
\text { - MIT: } 1 \mathrm{~W} / \mathrm{Kg}+1 \\
\mathrm{~W} / \mathrm{Kg}^{*} 3-\mathrm{min}^{-1} \text { until exhaustion } \\
1 \text { stage }=+1 \mathrm{~W} / \mathrm{Kg}\end{array}$ & $\begin{array}{l}\text { MIT } \\
- \\
1 \mathrm{~W} / \mathrm{Kg}+1 \\
\mathrm{~W} / \mathrm{Kg}^{*} 3-\mathrm{min}^{-1}\end{array}$ & 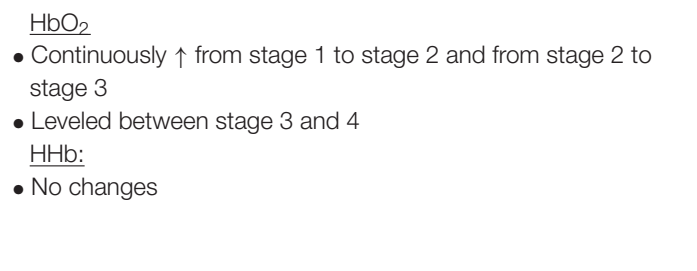 \\
\hline $\begin{array}{l}\text { Oussaidene } \\
\text { et al. (2015) }\end{array}$ & $\begin{array}{l}\text { Endurance athletes } \\
\text { (TR) } \\
13 \mathrm{M} \\
\text { A: } 26 \pm 5 \text { year } \\
\text { Length: } \\
178.5 \pm 5.8 \mathrm{~cm} \\
\text { Mass: } 70 \pm 6 \mathrm{~kg} \\
\text { Training status: PL3 } \\
\text { Untrained (UNT) } \\
11 \mathrm{M} \\
\text { A: } 24 \pm 6 \text { year } \\
\text { Length: } \\
179.7 \pm 4.9 \mathrm{~cm} \\
\text { Mass: } 77.2 \pm 6.1 \\
\text { kg } \\
\text { Training status: PL1 }\end{array}$ & $\begin{array}{l}\text { Oxymon, Artinis, Zetten, The } \\
\text { Netherlands } \\
\text { IOD: } 50 \mathrm{~mm} \\
\text { Position: Fp1 and F3, according to the } \\
\text { modified international EEG 10-20 } \\
\text { system } \\
780 \text { and } 857 \mathrm{~nm} \\
\text { Continuous wave }\end{array}$ & $\begin{array}{l}\text { (Cycling) } \\
\text { - } 5 \text { min resting period } \\
\text { - } 3 \text {-min warm-up } 60 \mathrm{~W} \text { (UNT), } \\
100 \mathrm{~W}(\mathrm{TR}) \\
\text { - MIT: warm-up workload } \uparrow \text { with } \\
1 \mathrm{~W} / 3 \text {-s until exhaustion } \\
\text { - } 8 \text {-min rest (2 active, } 8 \text { passive) } \\
\text { - TTE: } \\
\left.\text { o } 1 \text {-min (50 W } \text { max }_{\text {max }}\right) \\
\text { o } 105 \% \mathrm{~W}_{\max } \text { until exhaustion }\end{array}$ & $\begin{array}{l}\text { MIT } \\
- \\
60 \mathrm{~W} \text { or } 100+1 \mathrm{~W} / 3-\mathrm{s} \\
\text { TTE } \\
- \\
105 \% \mathrm{~W}_{\max }\end{array}$ & 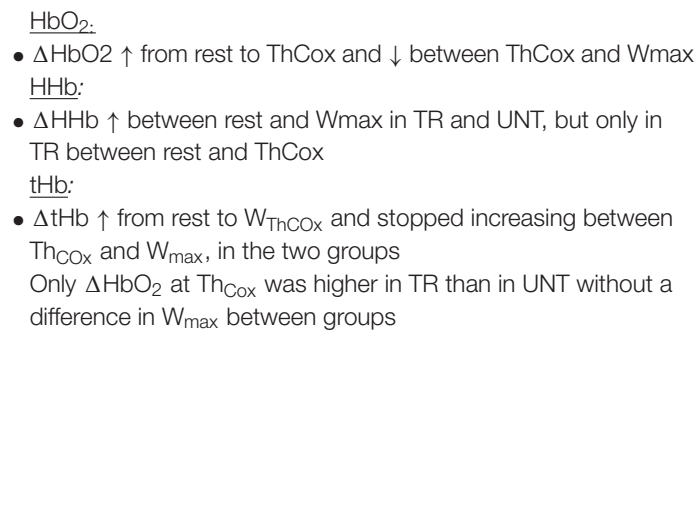 \\
\hline $\begin{array}{l}\text { Tempest and } \\
\text { Parfitt (2016) }\end{array}$ & $\begin{array}{l}\text { High tolerance }(\mathrm{HT}) \\
7 \mathrm{M}, 7 \mathrm{~F} \\
\text { A: } 20.6 \pm 1.4 \text { year } \\
\text { Length: } \\
171.0 \pm 7.5 \mathrm{~cm} \\
\text { Mass: } \\
69.3 \pm 7.3 \mathrm{~kg} \\
\text { BMl: } 23.4 \pm 2.0 \\
\text { Training status: PL2 } \\
\text { Low tolerance (LT) } \\
7 \mathrm{M}, 7 \mathrm{~F} \\
\text { A: } 21.5 \pm 3.4 \text { year } \\
\text { Length: } \\
173.2 \pm 9.1 \mathrm{~cm} \\
\text { Mass: } \\
68.6 \pm 12.8 \mathrm{~kg} \\
\text { BMl: } 22.4 \pm 2.7 \\
\text { Training status: PL1 }\end{array}$ & $\begin{array}{l}\text { NIRO } 200 \text { Hamamatsu Photonics, } \\
\text { Hamamatsu, Japan } \\
\text { IOD: } 40 \text { mm } \\
\text { Position: between Fp1 and F3 (left side) } \\
\text { and Fp2 and F4 (right side) of the } \\
\text { International 10-20 system }\end{array}$ & $\begin{array}{l}\text { (Cycling) } \\
\text { - MIT: } 20 \text { W/ min until exhaustion } \\
70 \text { RPM }\end{array}$ & $\begin{array}{l}\text { MIT } \\
- \\
\uparrow 20 \mathrm{~W} / \mathrm{min}\end{array}$ & 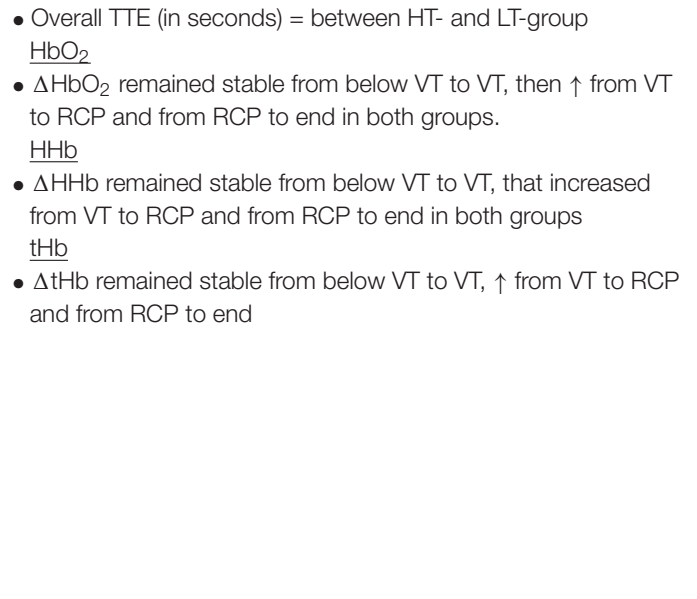 \\
\hline
\end{tabular}


TABLE 5B | (Continued)

\begin{tabular}{|c|c|c|c|c|c|}
\hline Study & $\begin{array}{l}\text { Sample } \\
\text { characteristics } \\
\text { (Mean } \pm \text { SD) }\end{array}$ & $\begin{array}{l}\text { NIRS-devise, Inter Optode } \\
\text { Distance (IOD), position, wave } \\
\text { specifications }\end{array}$ & Physical task & $\begin{array}{l}\text { Type of task and } \\
\text { exercise intensity }\end{array}$ & Outcome \\
\hline $\begin{array}{l}\text { Tempest et al. } \\
\text { (2017) }\end{array}$ & $\begin{array}{l}6 \mathrm{M}, 6 \mathrm{~F} \\
\text { A: } 26.2 \pm 3.0 \text { year } \\
\text { Length: } \\
173.7 \pm 7.6 \mathrm{~cm} \\
\text { Mass: } \\
72.7 \pm 9.1 \mathrm{~kg} \\
\text { Training status: PL1 }\end{array}$ & $\begin{array}{l}\text { Oxymon Mk II, Artinis Medical Systems, } \\
\text { Zetten, the Netherlands } \\
\text { IOD: } 38 \mathrm{~mm} \\
\text { Position: 10/20 international system for } \\
\text { electrode placement (the most inferior } \\
\text { probes in line with Fpz) }\end{array}$ & $\begin{array}{l}\text { (Cycling) } \\
\text { - MIT: } 20 \mathrm{~W} / \text { min until exhaustion. } \\
\text { Upright, recumbent and } \\
\text { semi-recumbent position. }\end{array}$ & $\begin{array}{l}\text { MIT } \\
- \\
20 \mathrm{~W} / \mathrm{min}\end{array}$ & 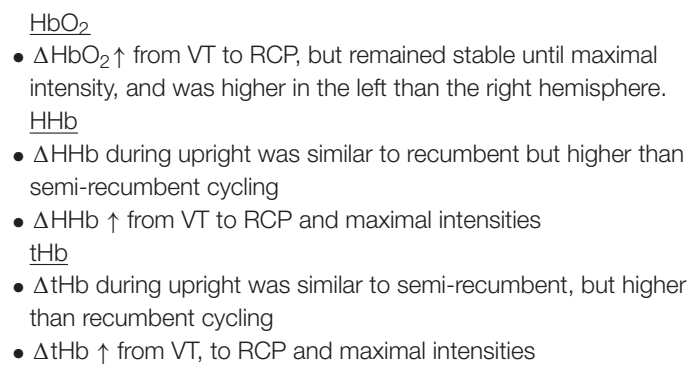 \\
\hline $\begin{array}{l}\text { Stevens et al. } \\
\text { (2018) }\end{array}$ & $\begin{array}{l}15 \mathrm{M} \\
\text { A: } 27.8 \pm 3.21 \text { year } \\
\text { Length: } \\
173.7 \pm 7.6 \mathrm{~cm} \\
\mathrm{BMl}: 24.96 \pm 3.21 \\
\mathrm{VO}_{2} \text { max: } \\
41.80 \pm 7.69 \mathrm{ml}^{\prime} \mathrm{kg}^{*} \mathrm{~min}^{-} \\
\text {Training status: } \mathrm{PL} 1\end{array}$ & $\begin{array}{l}\text { OxiplexTS, ISS, Champaign, Illinois, } \\
\text { Unite d States } \\
\text { IOD: 20, 25, 30, and } 35 \mathrm{~mm} \\
\text { Position: Fp1 and F2, according to the } \\
\text { international EEG 10-20 system } \\
690 \text { and } 830 \mathrm{~nm} \\
1^{-1}\end{array}$ & $\begin{array}{l}\text { (Cycling) } \\
\text {-2-min pre-exercise rest } \\
\text {-2-min resistance-free cycling at } \\
\text { a self-selected cadence } \\
30 \mathrm{~W} \text { increments every } 2 \text { min } \\
\text { until exhaustion }\end{array}$ & $\begin{array}{l}\text { MIT } \\
- \\
0+30 \text { W/2-min }\end{array}$ & $\begin{array}{l}\text { - } \mathrm{HbO}_{2} \text { and } \mathrm{tHb} \text { : little change up to } 15 \% \uparrow \mathrm{VO}_{2 \max } \text {, quadratic } \\
\text { increase up to } 75 \% \text { and a small increase above the } \mathrm{RCT} \\
\text { - } \mathrm{HHb} \text { : linear trend against exercise intensity } \\
\mathrm{COx} \text { was unchanged with intensity }\end{array}$ \\
\hline $\begin{array}{l}\text { Kojima et al. } \\
(2020)\end{array}$ & $\begin{array}{l}4 \mathrm{M}, 8 \mathrm{~F} \\
\text { A: } 21.6 \pm 0.2 \text { year } \\
\text { Length: } 162.6 \pm 2 \\
\text { cm } \\
\text { Mass: } \\
57.3 \pm 2.9 \mathrm{~kg} \\
\text { Training status: / }\end{array}$ & $\begin{array}{l}\text { OMM3000, Shimadzu Co., Kyoto, } \\
\text { Japan } \\
\text { IOD: } 30 \mathrm{~mm} \\
\text { Position: Cz position of the International } \\
\text { 10-20 System }\end{array}$ & $\begin{array}{l}\text { (Cycling) } \\
\text { 4-min pre-exercise rest } \\
\text { - 4-min warm-up } \\
20 \text { W increments/min until } \\
\text { exhaustion }\end{array}$ & $\begin{array}{l}\text { MIT } \\
- \\
0+20 \mathrm{~W} / \mathrm{min}\end{array}$ & $\begin{array}{l}\text { - } \mathrm{HbO}_{2} \uparrow \text { until RCP and } \downarrow \text { until exhaustion } \\
\text { - } \mathrm{HHb} \uparrow \mathrm{VT} \text { until exhaustion but not from rest to VT } \\
\text { - } \mathrm{tHb} \uparrow \text { from start to RCP and further to exhaustion } \\
\text { - } \mathrm{SBF} \uparrow \text { at } \mathrm{VT}, \mathrm{RCP} \text { and exhaustion compared to rest }\end{array}$ \\
\hline $\begin{array}{l}\text { Kojima et al. } \\
(2021)\end{array}$ & $\begin{array}{l}24 \mathrm{M} \\
\text { A: } 20.7 \pm 0 \text { year } \\
\text { Length: } \\
172.6 \pm 5.9 \mathrm{~cm} \\
\text { Mass: } \\
64.8 \pm 9.9 \mathrm{~kg} \\
\text { Training status: / }\end{array}$ & $\begin{array}{l}\text { LABNIRS, Shimadzu Co., Kyoto, Japan } \\
\text { IOD: } 30 \mathrm{~mm} \\
\text { Position: Cz position of the International } \\
\text { 10-20 System }\end{array}$ & $\begin{array}{l}\text { (Cycling) } \\
\text { 4-min pre-exercise rest } \\
\text {-4-min warm-up } \\
20 \text { W increments/min until } \\
\text { exhaustion }\end{array}$ & $\begin{array}{l}\text { MIT } \\
- \\
0+20 \mathrm{~W} / \mathrm{min}\end{array}$ & $\begin{array}{l}\text { - } \mathrm{HbO}_{2} \uparrow \text { until } \mathrm{RCP} \text { and } \downarrow \text { until exhaustion } \\
\text { - } \mathrm{RCP} \text { correlated with the } \mathrm{HbO}_{2} \text { decreasing point } \\
\text { } \uparrow \Delta \mathrm{HH} \mathrm{H}, \Delta \mathrm{THb}, \Delta \mathrm{CBV} \text {, and } \Delta \mathrm{SBF} \uparrow \text { with load until exhaustion } \\
\text { - } \Delta \mathrm{P}_{\mathrm{ET}} \mathrm{CO}_{2} \text { first } \uparrow \text { with incremental load and then } \downarrow \text { from } 60 \text { to } \\
100 \% \\
\text { - } \mathrm{P}_{\mathrm{ET}} \mathrm{CO}_{2} \text { correlated with } \Delta \mathrm{HHb} \text { but not with } \mathrm{HbO}_{2} \text { and } \Delta \mathrm{CBV}\end{array}$ \\
\hline
\end{tabular}

M, man; F, female; A, age; Y, Years BMI, Body Mass Index; Fat\%, body fat; KG, kilogram; Cm, Centimeter; mm, Millimeter; VO 2 peak, VO 2 max Maximal Oxygen Uptake; PL, Performance Level; NIRS, Near Infrared Spectroscopy; IOD, Inter Optode Distance; TTE, Time To Exhaustion; $\mathrm{SaO}_{2}$, Arterial Oxygen Saturation; $\mathrm{VO}_{2}$, Oxygen Uptake, $V_{\mathrm{E}}$, Ventilation; HR, Heart Rate; tHb, Total Hemoglobin; HbO 2 , Oxygenated hemoglobin; HHb, Deoxygenated Hemoglobin; MRI, Magnetic Resonance Imaging; Sec, Second; Min, Minute; Km, Kilometer; H, Hour; BP, Blood Pressure; PFC, Prefrontal Cortex; PMC, Premotor Cortex; m-SMC, Medial SensoriMotor Cortex: TT. Time-Trial: EEG, Electroencephalography: RPE, Rating of Perceived Exertion; CPT(RM, Constant-Power Test (Respiratory Maneuver): RPM, Rotations per Minute: SBF Skin Blood Flow: TOl, Tiscue Oxygenation Index; MVC, Maximal Voluntary Contraction; MIT, Maximal Incremental Exercise Test; Cox, Cerebral Oxygenation; FIT, Fatigue Training Test; PER, Post-Exercise Rest; MAP, Mean Arterial Pressure; PAP, Peak Aerobic Power; T, Trial; $\mathrm{P}_{\mathrm{ET}} \mathrm{CO}_{2}$, End-tidal pressure of $\mathrm{CO}_{2}$. 
Kojima et al. (2021) reported a relationship between the point of decline in $\left[\mathrm{HbO}_{2}\right]$ and RCP. Kojima et al. (2021) also reported that end-tidal $\mathrm{CO}_{2}$ was decreased by respiratory compensation after RCP and that end-tidal $\mathrm{CO}_{2}$ was associated with $\mathrm{HHb}$.

Data in relation to $\mathrm{HHb}$ were inconsistent between studies. Timinkul et al. (2008) and Kojima et al. (2020) reported that $\mathrm{HHb}$ slightly decreased from the start of the trial until the cerebral blood volume threshold, remained stable until the RCP, and thereafter increased until exhaustion. Rupp and Perrey (2008) and Oussaidene et al. (2015), however, reported that $\mathrm{HHb}$ increased from a warm-up until exhaustion. Rupp and Perrey (2008) and Oussaidene et al. (2015) also reported that $\mathrm{tHb}$ concentrations increased between rest, VT2, and ThCox, after which it was stabilized until exhaustion. Three studies acknowledged the initial increase in $\left[\mathrm{HbO}_{2}\right]$ from the start of exercise, but instead of a subsequent decrease in $\left[\mathrm{HbO}_{2}\right]$, a steady state was reached (Tempest et al., 2014, 2017; Jung et al., 2015). Two studies reported an increase in $\left[\mathrm{HbO}_{2}\right]$ from the start of an exercise until RCP, after which $\left[\mathrm{HbO}_{2}\right]$ remained stable until exhaustion (Tempest et al., 2014, 2017; Jung et al., 2015). Tempest et al. (2014) reported that $\left[\mathrm{HbO}_{2}\right]$ increases from the value below VT $\left(54.5 \pm 4.1 \% \mathrm{VO}_{2 \max }\right)$ until RCP $\left(90.2 \pm 4.9 \% \mathrm{VO}_{2 \max }\right)$ and remains stable from RCP until exhaustion. The authors also showed that [HHb] remains stable between the value below VT and VT $(68.2 \pm 4.5 \%$ $\mathrm{VO}_{2 \mathrm{max}}$ ) and increases from VT until exhaustion, whereas [tHb] continuously increases from the value below VT until exhaustion. Tempest et al. (2017) found that [HHb] increased from VT until exhaustion, whereas $\left[\mathrm{HbO}_{2}\right]$ remained stable between the start of exercise and VT $\left(67.2 \pm 2.9 \% \mathrm{VO}_{2 \max }\right)$, increased between VT and RCP $\left(87.9 \pm 3.2 \% \mathrm{VO}_{2 \max }\right)$, and thereafter remained stable until maximal intensity. Additionally, the results of the study by Tempest et al. (2014, 2017) were supported by Jung et al. (2015) who found a continuous increase in PFC oxygenation from stage 1 to stage 3 and stabilized $\mathrm{HbO}_{2}$ concentrations between stages 3 and 4 during a multistage protocol that started at $1 \mathrm{~W} / \mathrm{kg}$ (stage 1 ) and increased with 1 $\mathrm{W} / \mathrm{kg}$ every stage. In contrast to Tempest et al. (2014, 2017) and Jung et al. (2015) found no significant differences in [HHb] between stages/intensities. Finally, two studies reported increases in $\left[\mathrm{HbO}_{2}\right]$ from RCP until exhaustion. Tempest and Parfitt (2016) measured prefrontal oxygenation during a maximal incremental test (MIT) between groups with high- and lowself-reported tolerance to exercise. The data indicated that $\left[\mathrm{HbO}_{2}\right]$ remains stable between the value below VT and VT $\left(\right.$ high $=49.8 \pm 4.8 \% \mathrm{VO}_{2 \max }$, low $\left.=54.6 \pm 5.0 \% \mathrm{VO}_{2 \max }\right)$ and increased between VT and RCP VT (high $=76.3 \pm 5.1 \%$ $\mathrm{VO}_{2 \max }$, low $\left.=84.1 \pm 5.9 \% \mathrm{VO}_{2 \max }\right)$ and from $\mathrm{RCP}$ to exhaustion. According to Tempest et al. (2014, 2017), [HHb] and $[\mathrm{tHb}$ ] remained stable from the value below VT to VT, increased from VT to RCP, and also increased from RCP to exhaustion in both tolerance groups. In contrast, Stevens et al. (2018) reported a small increase in $\left[\mathrm{HbO}_{2}\right],[\mathrm{HHb}]$, and [tHb] up to $15 \% \mathrm{VO}_{2 \mathrm{max}}$, followed by a quadratic increase up to $75 \% \mathrm{VO}_{2 \max }$ and a smaller increase above the value of RCT. Importantly, $\left[\mathrm{HbO}_{2}\right],[\mathrm{HHb}],[\mathrm{tHb}]$ increases were most prominent at workloads near RCP (Stevens et al., 2018). A visual representation of $\mathrm{HbO}_{2}$ and $\mathrm{HHb}$ during MIT can be found in Table 6.

\section{Prefrontal Cortex Oxygenation During Whole-Body Endurance Tasks}

Seventeen studies measured TTE, TT, a constant load with fixed intensity- and adaptive walking/running-exercise protocols. Eleven of the included studies assessed PFC oxygenation during whole-body endurance protocols with a constant intensity and fixed duration (Ide et al., 1999; Fumoto et al., 2010; Miyazawa et al., 2013; Rupp et al., 2013; Giles et al., 2014; Tsubaki et al., 2016, 2018; Radel et al., 2017; Takehara et al., 2017; Hiura et al., 2018; Ohyanagi et al., 2018). In these studies, participants were asked to complete an endurance cycling task at a certain predetermined intensity (e.g., \%HRmax and $\% \mathrm{VO}_{2 \text { peak }}$ ) for a predetermined time. We classify these studies according to the following intensities: (1) light to moderate, (2) vigorous, (3) near-to-maximal, and (4) supramaximal.

Light to moderate intensity: two studies assessed PFC during 10 min of cycling at 30 and $50 \% \mathrm{VO}_{2 \text { peak }}$ (Takehara et al., 2017) and 30 and $60 \% \mathrm{VO}_{2 \max }$ (Ide et al., 1999). Ide et al. (1999) found an increase in $\left[\mathrm{HbO}_{2}\right],[\mathrm{HHb}]$, and $[\mathrm{tHb}]$ in proportion to work rate, at the intensities of 30 and $60 \% \mathrm{VO}_{2 \max }$. Increases were significantly higher at $60 \% \mathrm{VO}_{2 \mathrm{max}}$, and $\left[\mathrm{HbO}_{2}\right]$ reached a maximal level during the first few minutes of recovery. These data were replicated by Takehara et al. (2017) who reported an increase in $\left[\mathrm{HbO}_{2}\right]$ between 300 and $600 \mathrm{~s}$, which was higher at 50\% $\mathrm{VO}_{2 \text { peak }}$ than $30 \% \mathrm{VO}_{2 \text { peak, }}$ but reported a decrease in $\left[\mathrm{HbO}_{2}\right]$ between 30 and $300 \mathrm{~s}$. During a 4-min facial cooling intervention to reduce prefrontal skin blood flow and $\left[\mathrm{HbO}_{2}\right]$, Miyazawa et al. (2013) reported that prefrontal $\left[\mathrm{HbO}_{2}\right]$ increased during an 11-min cycling exercise at $60 \% \mathrm{HRmax}$ (light intensity). The authors reported that neither the light-intensity cycling exercise nor the facial cooling intervention had an influence on [HHb]. The changes in $[\mathrm{tHb}]$ were similar to the changes in $\left[\mathrm{HbO}_{2}\right]$ and were related to prefrontal skin blood flow (Miyazawa et al., 2013). In a 15 -min cycling task at $30 \% \mathrm{HR}$ reserve (light intensity), Hiura et al. (2018) reported that $\left[\mathrm{HbO}_{2}\right]$ remained stable during the first $5 \mathrm{~min}$ but increased during the last $2 \mathrm{~min}$ of the trial and that $\mathrm{HHb}$ remained unchanged during the entirety. Hiura et al. (2018) also monitored the prefrontal blood flow and partial pressure of $\mathrm{CO}_{2}\left(\mathrm{PCO}_{2}\right)$ and reported that cerebral blood flow $(\mathrm{CBF})$ and $\mathrm{PCO}_{2}$ increased during the first $5 \mathrm{~min}$ and remained unchanged during the last $2 \mathrm{~min}$. The results of the study by Ide et al. (1999) were replicated by Fumoto et al. (2010) who found a gradual increase in $\left[\mathrm{HbO}_{2}\right]$ during a 15 -min cycling task at an intensity equaling 12-13 on the RPE scale (Borg, 1998) (moderate intensity), after which $\left[\mathrm{HbO}_{2}\right]$ reached a steady state until the end. Only a small decrease in $[\mathrm{HHb}$ ] was found throughout the trial (Fumoto et al., 2010). A gradual increase in $\left[\mathrm{HbO}_{2}\right]$ during moderate-intensity exercise was supported by Tsubaki et al. (2018) who reported that $\left[\mathrm{HbO}_{2}\right]$ continually increased in right $\mathrm{PFC}$ during a 20-min cycling exercise trial at an intensity of $50 \% \mathrm{VO}_{2 \text { peak }}$. Prefrontal skin blood flow also increased over the time of the trial and decreased again during the postexercise rest (Tsubaki et al., 2018). In a 20-min moderate-intensity (50\% 
TABLE 6 | Summary of finding on the evolution of $\mathrm{HbO}_{2}$ and $\mathrm{HHb}$ during MIT.

\begin{tabular}{|c|c|c|c|c|c|c|}
\hline \multirow[b]{2}{*}{ Study } & \multicolumn{3}{|c|}{$\mathrm{HbO}_{2}$} & \multicolumn{3}{|c|}{$\mathrm{HHb}$} \\
\hline & Start-VT & VT-RCP & RCP-exhaustion & Start-VT & VT-RCP & RCP-exhaustion \\
\hline Bhambhani et al. (2007) & $\uparrow$ & $\uparrow$ & $\downarrow$ & NA & NA & NA \\
\hline Timinkul et al. (2008) & $\uparrow$ & $\uparrow$ & $\downarrow$ & $\downarrow$ & $=$ & $\uparrow$ \\
\hline Tempest et al. (2014) & $\uparrow$ & $\uparrow$ & $=$ & $=$ & $\uparrow$ & $\uparrow$ \\
\hline Jung et al. (2015) & $\uparrow$ & $\uparrow$ & $=$ & $=$ & $=$ & $=$ \\
\hline Oussaidene et al. (2015) & $\uparrow$ & $\uparrow$ & $\downarrow$ & $\uparrow$ & $\uparrow$ & $\uparrow$ \\
\hline Tempest and Parfitt (2016) & $=$ & $\uparrow$ & $\uparrow$ & $=$ & $\uparrow$ & $\uparrow$ \\
\hline Tempest et al. (2017) & $=$ & $\uparrow$ & $=$ & $=$ & $\uparrow$ & $\uparrow$ \\
\hline Stevens et al. (2018) & $\uparrow$ & $\uparrow$ & $\uparrow$ & $\uparrow$ & $\uparrow$ & $\uparrow$ \\
\hline Kojima et al. (2020) & $\uparrow$ & $\uparrow$ & $\downarrow$ & $\downarrow$ & $=$ & $\uparrow$ \\
\hline Kojima et al. (2021) & $\uparrow$ & $\uparrow$ & $\downarrow$ & $\uparrow$ & $\uparrow$ & $\uparrow$ \\
\hline
\end{tabular}

$\mathrm{VO}_{2 \max }$ ) cycling trial, an increase in $\left[\mathrm{HbO}_{2}\right]$ was found between 3 and 6 min after which $\mathrm{HbO}_{2}$ levels remained stable until the end (Ohyanagi et al., 2018). Within this research, prefrontal skin blood flow increased gradually throughout the trial, supporting the findings of Miyazawa et al. (2013) and Tsubaki et al. (2018). Finally, in two separate 90-min moderate-intensity cycling tests at $58-60 \% \mathrm{VO}_{2 \text { peak, }}$, at the cadence of 40 and $80 \mathrm{RPM}$, it was found that at the end of the exercise, $[\mathrm{tHb}]$ and $\left[\mathrm{HbO}_{2}\right]$ values were lower at $80 \mathrm{RPM}$ than at $40 \mathrm{RPM}$, and [HHb] was lower at 80 RPM but remained unchanged at 40 RPM.

Suzuki et al. (2004) measured PFC after performing a 90s locomotor task at three different speeds $(3,6$, and $9 \mathrm{~km} / \mathrm{h}$ ), each with a 30-s rest. Before the start of the task, $\left[\mathrm{HbO}_{2}\right]$ and [tHb] increased, which was most prominent at $9 \mathrm{~km} / \mathrm{h}$, and peaked prior to the treadmill speed steadying. After reaching a constant speed, $\left[\mathrm{HbO}_{2}\right]$ and $[\mathrm{tHb}]$ decreased and returned to baseline, or lower than baseline, during the rest of the task. After stopping locomotion, temporal drops in $\left[\mathrm{HbO}_{2}\right]$ were seen before returning to baseline. $\mathrm{PFC}$ activation was greater, and $\mathrm{HbO}_{2}$ was higher at $9 \mathrm{~km} / \mathrm{h}$ than 3 and $6 \mathrm{~km} / \mathrm{h}$. No differences were shown between and 3 and $6 \mathrm{~km} / \mathrm{h}$ (Suzuki et al., 2004).

Vigorous intensity: two studies examined the effects of vigorous cycling exercise on prefrontal oxygenation (Rupp et al., 2013; Giles et al., 2014). Rupp et al. (2013) asked participants to perform three consecutive 80 -min cycling trials at $45 \%$ peak aerobic power output, each separated by 25 min of neuromuscular function testing. An increase in $\left[\mathrm{HbO}_{2}\right]$ and $[\mathrm{tHb}]$ was found in trial 1 , reaching a plateau after approximately $40 \mathrm{~min}$, whereas [HHb] progressively increased between 20 and $80 \mathrm{~min}$ in trials 1, 2, and 3 . [HHb] was significantly lower in trials 2 and 3 than trial 1. In a sample of regular exercisers, Giles et al. (2014) found that $\left[\mathrm{HbO}_{2}\right],[\mathrm{HHb}]$, and $[\mathrm{tHb}]$ increased as a result of exercise intensity. Participants completed three separate 30-min cycling trials at $52 \%$ (low intensity), 68\% (moderate), and $84 \%$ (vigorous) HRmax, respectively (Giles et al., 2014). Similar $\mathrm{HbO}_{2}$ values were found during the first $15 \mathrm{~min}$ among exercise intensities. After $18 \mathrm{~min}, \mathrm{HbO}_{2}$ was significantly higher at a moderate and high intensity than low intensity, and significantly higher at a vigorous than moderate intensity. Higher values of [HHb] were reported as a result of exercise intensity with higher values of
[HHb] at a vigorous than moderate intensity, which was also higher than a low intensity (Giles et al., 2014). HHb values were steady until min 12 after which the values increased over time to min 23. From this point, $\mathrm{HHb}$ increased according to exercise intensity, with greater increases in $[\mathrm{HHb}]$ at a vigorous than moderate intensity, which in turn was higher than low intensity. [tHb] increased with each minute from the start and was similar between intensities until min 16, after which [tHb] increased between all intensities.

Near-to-maximal intensity: Two studies reported similar results in PFC during a 5-km running TT. Billaut et al. (2010) reported increased prefrontal $\left[\mathrm{HbO}_{2}\right],[\mathrm{HHb}],[\mathrm{tHb}]$, and $\mathrm{CBV}$ values from the start until a $2.5-\mathrm{km}$ point, from which these values remained constant until $\mathrm{km} 4.5$ and displayed evidence of deoxygenation in the last $0.5 \mathrm{~km}$. Regional blood flow remained stable throughout this. Santos-Concejero et al. (2015) described increases in $\left[\mathrm{HbO}_{2}\right]$ and $[\mathrm{HHb}]$ over the first half of the trial, steady $\mathrm{HbO}_{2}$ values in the second half of the trial, and further increases in $[\mathrm{HHb}]$ until completion. Additionally, TOI reduced over the first $1.5 \mathrm{~km}$, after which it remained stable until the end. However, in contrast to Billaut et al. (2010), [tHb] remained stable for the first half of the trial and increased progressively from $3 \mathrm{~km}$ until completion. Pires et al. (2016), used a 4-km cycling TT and found that prefrontal $\left[\mathrm{HbO}_{2}\right],[\mathrm{HHb}]$, and $[\mathrm{tHb}]$ increased until $70 \%$ of the TT and decreased upon completion. Pires et al. (2016) also assessed prefrontal oxygenation during a preliminary MIT (100 W $+25 \mathrm{~W} / \mathrm{min}$, until exhaustion), where prefrontal $\left[\mathrm{HbO}_{2}\right],[\mathrm{HHb}]$, and $[\mathrm{tHb}]$ increased until $70 \%$ of the exercise, and thereafter decreased. Santos-Concejero et al. (2017) used a fatigue training test consisting of continuous 1$\mathrm{km}$ repeated running trials, with 30 -s recovery until exhaustion, at a pace $5 \%$ faster than their $5-\mathrm{km}$ running TT (completed 2 days earlier). During the test, $\triangle\left[\mathrm{HbO}_{2}\right]$ was elevated compared to baseline throughout the test, decreased at the end of each repetition, and progressively decreased in the first, fifth, and final repetition. $\Delta[\mathrm{HHb}]$ increased over the course of the test with increases during each running bout and decreases during each 30-s recovery period until completion. A decline in cerebral oxygen was negatively associated with the speed at which the test was completed. Radel et al. (2017) examined hemodynamic 
changes when coping with the expectation of prolonged exercise. Participants were told that they would perform a cycling trial at an intensity equivalent to $60 \%$ peak aerobic power and that it would take 10 - or 60 -min according to the condition. In both experimental sessions, the trial was stopped after $10 \mathrm{~min}$. A main effect of time was found, whereby $\left[\mathrm{HbO}_{2}\right]$ was lower in the first period than in the second and third. Compared to the 10-min condition, in the 60-min condition, smaller $\left[\mathrm{HbO}_{2}\right]$ elevations at the right dorsomedial PFC and higher $\left[\mathrm{HbO}_{2}\right]$ elevations at the right dorsomedial PFC were reported. No differences in RPE were found between conditions.

Supramaximal intensity: Shibuya et al. (2004) studied the effect of a supramaximal TTE performance at $120 \%$ of participants' $\mathrm{VO}_{2 \text { peak }}$ on cerebral oxygenation. $\left[\mathrm{HbO}_{2}\right]$ increased during the first $30 \mathrm{~s}$ of exercise, after which $\left[\mathrm{HbO}_{2}\right]$ and $[\mathrm{tHb}]$ gradually decreased over time, resulting in significantly decreased values from the pre-exercise level. [HHb] remained constant throughout the test.

\section{DISCUSSION}

In this review, we outlined the current knowledge on the development of PFC oxygenation during whole-body endurance exercise through fNIRS. Research indicates that during MIT, $\mathrm{HbO}_{2}$ increases until RCP, after which it decreases until exhaustion or remains in a steady state. Regarding submaximal exercise, prefrontal oxygenation increases during light, moderate, and vigorous intensity workloads but reaches a steady state over time. According to the findings using MIT at a nearmaximal intensity, prefrontal oxygenation cannot be maintained, and deoxygenation occurs at PFC. RCP appears to act as a ThCox, with the occurrence of the deoxygenation of PFC when RCP exceeds. The findings presented in this review show no evidence for an increase in cerebral energy metabolism to be responsible for the deoxygenation of the PFC but instead indicate that an increase in respiratory ventilation in reaction exerciseinduced hypocapnia results in a decrease in $\mathrm{CBF}$ and thus [ $\mathrm{tHb}$ ], $\left[\mathrm{HbO}_{2}\right]$, and [HHb].

Quality assessment was scored as moderate to strong in most studies, with only three studies being scored as weak (Takehara et al., 2017; Ohyanagi et al., 2018; Kojima et al., 2020). Two major limitations regarding the quality of studies were: (1) the method of participants' selection or source information variables being insufficiently described, whereby participants are often described as "healthy volunteers", and (2) insufficient data being presented to assess if the sample size was appropriate. The majority (74\%) of the studies used observational study design and were therefore exempted from the subquestions E, F, G, and L of the quality assessment.

\section{Near-IR Spectroscopy Methodology}

As part of the inclusion criteria, all studies used NIRS to investigate PFC oxygenation. With this technique, near-IR light (NIR) is emitted through the scalp up to the neuronal tissue, where the light is either absorbed or scattered off. NIR with wavelengths between the optical window of 700 and $900 \mathrm{~nm}$ pass through most biological tissues, including bone because there is low absorption and scattering of photons (Ekkekakis, 2009). A body of research has shown that NIRS with wavelengths around $760 \mathrm{~nm}$ has an absorption peak within $\mathrm{HHb}$, whereas in $\mathrm{HbO}_{2}$ this is the case at $830 \mathrm{~nm}$, making $\mathrm{HHb}$ and $\mathrm{HbO}_{2}$ distinguishable from each other (Ekkekakis, 2009). In terms of NIRS device positioning, 23 studies used the international EEG $10-20$ or 10-5 system, which is recognized to describe the placement of the scalp electrodes. This is in accordance with the methodological review of Herold et al. (2018) who stated that the international EEG 10-20/10-5 system is the most common and practical strategy for optode placement and thereby ensures that the region of interest is targeted. Next to the optode placement, the IOD is an important factor as it determines the depth of the NIRS measurement. Of the included studies, 16 applied an IOD of between 30 and $40 \mathrm{~mm}$, which is appropriate for adults (Leon-Carrion and Leon-Dominguez, 2012; Herold et al., 2018). However, two studies used an IOD of $25 \mathrm{~mm}$ (Giles et al., 2014; Jung et al., 2015), which has been shown to include gray matter into the sample volume, and eight studies used an IOD of between 45 and 50 mm (Ide et al., 1999; Shibuya et al., 2004; Rupp and Perrey, 2008; Timinkul et al., 2008; Fumoto et al., 2010; Keramidas et al., 2011; Oussaidene et al., 2015; Pires et al., 2016), where the contribution of extracranial tissue is negligible (Leon-Carrion and Leon-Dominguez, 2012). Another effect of using a short channel (IOD $\leq 25 \mathrm{~mm}$ ) is that NIRS signals are influenced by skin blood flow and $\mathrm{CBF}$ due to a more superficial measurement (Matsukawa et al., 2015). A consensus is needed for the studies that use NIRS devices to allow appropriate comparisons between studies. The guidelines for the usage of NIRS can be found in Herold et al. (2018).

\section{Prefrontal Cortex Oxygenation During Incremental Exercise (Maximal Incremental Test)}

It was hypothesized that prefrontal oxygenation would increase during submaximal exercise and subsequently decrease at nearmaximal intensities. The results of this systematic review largely support this hypothesis but also reveal some new insights into central regulation during endurance performance. Rooks et al. (2010) concluded that cerebral oxygenation increases from lowto-vigorous intensities after which it reaches a plateau or declines toward baseline at near-to-maximal intensities. Rooks et al. (2010) focused on cerebral oxygenation in general, this review specifically focused on PFC and how oxygenation in this brain region evolves during whole-body endurance exercise. About 9 of 10 MIT studies found an increase in $\left[\mathrm{HbO}_{2}\right]$ during the first part of the incremental exercise. This initial increase can be explained by peripheral hemodynamic changes, such as increased cardiac output and metabolic demand in the function of the start of locomotion, and a gradual increase of intensity. Only in Tempest and Parfitt (2016) did $\left[\mathrm{HbO}_{2}\right]$ remain unchanged from the start until VT. All of the included studies reported an increase in $\left[\mathrm{HbO}_{2}\right]$ from VT until RCP, after which prefrontal oxygenation decreased (Bhambhani et al., 2007; Rupp and Perrey, 2008; Timinkul et al., 2008; Oussaidene et al., 2015; 
Kojima et al., 2020, 2021) or reached a plateau (Tempest et al., 2014; Jung et al., 2015; Tempest and Parfitt, 2016), supporting previous research (Rooks et al., 2010). Additionally, Kojima et al. (2021) reported that the point at which $\left[\mathrm{HbO}_{2}\right]$ declined was correlated with RCP. On the other hand, two studies found a further increase in $\left[\mathrm{HbO}_{2}\right]$ (Tempest and Parfitt, 2016; Stevens et al., 2018). In comparison with a steep increase from VT until RCP, Tempest and Parfitt (2016), did not declare or provide details on the degree of the increase in $\left[\mathrm{HbO}_{2}\right]$, whereas Stevens et al. (2018) reported only a small increase in $\left[\mathrm{HbO}_{2}\right]$ from RCP until exhaustion. These results together with the other seven studies show that RCP is a crucial point for prefrontal oxygenation as after this point prefrontal oxygenation endures significant changes. RCP can be defined as the point at which arterial $\mathrm{PCO}_{2}$ starts to decline during strenuous exercise and can be interpreted as a ventilatory response to maintain the acid-base balance by increasing ventilation (Rausch et al., 1991; van den Aardweg and de Groot, 2015).

Two studies (Timinkul et al., 2008; Kojima et al., 2021) measured end-tidal $\mathrm{CO}_{2}$ pressure during MIT. Timinkul et al. (2008) found that end-tidal $\mathrm{CO}_{2}$ pressure gradually increased with intensity and reached its peak at RCP, after which it gradually decreased until exhaustion. These results were supported by Kojima et al. (2021) who found an increase in end-tidal $\mathrm{CO}_{2}$ pressure with incremental load and a subsequent decrease from $60 \%$ to $100 \%$ of the MIT. This decrease was also correlated with $[\mathrm{HHb}]$ and $\mathrm{PCO}_{2}$. Research has shown that $\mathrm{PCO}_{2}$ has a direct impact on CBF (Lassen, 1959; Poulin et al., 1996; Yoon et al., 2012). The blood-brain barrier is permeable to $\mathrm{CO}_{2}$ but retains $\left[\mathrm{H}^{+}\right]$and $\left[\mathrm{HCO}_{3}{ }^{-}\right]$ions, making $\mathrm{CO}_{2}$ an important respiratory stimulant to the central chemoreceptors (Ainslie et al., 2007; Galán-Rioja et al., 2020). The brain requires a constant CBF within a narrow range of 60 and $150 \mathrm{mmHg}$ despite changes in mean arterial pressure (MAP) (Ogoh and Ainslie, 2009). This regulatory mechanism is called cerebral autoregulation (Ogoh and Ainslie, 2009). Hyperventilation caused by intense exercise subsequently results in a drop in $\mathrm{PCO}_{2}$. The state of low $\mathrm{PCO}_{2}$ in the arterial blood, also referred to as hypocapnia, causes cerebral vasoconstriction, a cerebral autoregulatory mechanism, which results in a decrease in CBF (Ogoh and Ainslie, 2009). Nybo and Rasmussen (2007) stated that when CBF and cerebral oxygenation fall below a critical level, the motor output cannot be maintained. These results have been demonstrated in several studies that prolonged aerobic exercise was improved by increasing cerebral oxygenation through additional inspired $\mathrm{O}_{2}$ levels (Nielsen et al., 1999; Subudhi et al., 2007). Besides the association between $\left[\mathrm{HbO}_{2}\right]$ and RCP, Kojima et al. (2021) found that end-tidal $\mathrm{CO}_{2}$ decreases after RCP. However, no correlation between end-tidal $\mathrm{CO}_{2}, \mathrm{CBF}$, and cerebral $\mathrm{O}_{2}$ exchange was reported. Kojima et al. (2021) suggests that a decrease in $\left[\mathrm{HbO}_{2}\right]$ and an increase in $[\mathrm{HHb}]$ before exhaustion during MIT are related to cerebral $\mathrm{O}_{2}$ metabolism by a neural activity increase. While this is an interesting suggestion, it must be cautioned as cerebral blood volume and cerebral oxygen exchange are only the estimated values. Even though NIRS is not capable of measuring blood flow, a decrease in $\mathrm{CBF}$ through $\mathrm{PCO}_{2}$ and cerebral autoregulation is a promising hypothesis to help explain changes in prefrontal oxygenation at RCP. However, there is a lack of research to objectively assess both cerebral oxygenation and CBF simultaneously. Studies combining NIRS with $\mathrm{CBF}$ velocity measurement techniques, such as transcranial doppler (TCD) ultrasonography and PET, could help elucidate the mediators of prefrontal oxygenation during exercise and the role of RCP as a ThCox.

\section{Prefrontal Cortex Oxygenation During Whole-Body Endurance Tasks}

This study is the first review to collect empirical evidence on PFC oxygenation during non-incremental whole-body endurance exercise. It was hypothesized that prefrontal oxygenation would increase during prolonged submaximal exercise until a steady state is reached. Additionally, we hypothesized that during nearmaximal intensities PFC oxygenation cannot be maintained, resulting in a decrease near the exhaustion or end of the exercise. Maximal incremental studies have shown that PFC oxygenation is sensitive to exercise intensity. As a result, we have subdivided this section into light-to-moderate, vigorous, and near-to-maximal intensity.

\section{Light-to-Moderate Intensity}

Three low-intensity protocol studies found an increase in $\left[\mathrm{HbO}_{2}\right]$ from the start to the end of exercise (Ide et al., 1999; Miyazawa et al., 2013; Takehara et al., 2017). Although in the study of Takehara et al. (2017) an initial decrease in $\left[\mathrm{HbO}_{2}\right]$ was found between the start and $3 \mathrm{~min}$ into the cycling trial, this can be explained given that $\mathrm{O}_{2}$-supply does not match the $\mathrm{O}_{2}$ demand at the beginning of exercise (McArdle et al., 2010). Miyazawa et al. (2013) supported these findings and found a correlation between TOI, $\mathrm{HbO}_{2}$, and $\mathrm{tHb}$ with prefrontal skin blood flow. This triggers the question of whether $\mathrm{HbO}_{2}$ was measured at PFC and not superficially at the skin of the scalp, as vasoconstriction through cooling results in a decreased skin blood flow. Alternatively, $\mathrm{HHb}$ was not correlated with skin blood flow as $\mathrm{HHb}$ was not affected by the face cooling intervention, nor by an 11-min cycling task. Importantly, Miyazawa et al. (2013) implemented a 4-min incremental warmup until reaching the intensity of $60 \%$ HRmax. This can help explain why $\mathrm{HHb}$ was not changed throughout the 11-min cycling exercise as several studies have pointed out that $\mathrm{HHb}$ is correlated with exercise intensity (Rupp and Perrey, 2008; Stevens et al., 2018; Kojima et al., 2021). Only one study objectively measured prefrontal blood flow and end-tidal $\mathrm{CO}_{2}$ during light-intensity exercise (Hiura et al., 2018). The authors reported that the prefrontal $\mathrm{CBF}$ and end-tidal pressure of $\mathrm{CO}_{2}$ initially increased but remained unchanged during the last few minutes of exercise. Similarly, the initial decrease in $\left[\mathrm{HbO}_{2}\right]$ was simultaneously associated with a steep increase in end-tidal $\mathrm{CO}_{2}$, which can be explained though $\mathrm{O}_{2}$ deficit at the start of the exercise. However, it is important to note that this study used both NIRS and PET to measure oxygenation and CBF, respectively, in two different samples. It is therefore unclear whether the results would be similar in the case of measurement at the same time point in the same person. Future research is 
needed to examine whether these results will be the same when oxygenation and CBF are measured in the same sample.

Regarding moderate intensity endurance tasks, prefrontal oxygenation gradually increases in $\left[\mathrm{HbO}_{2}\right]$ over time until a steady state is reached. Ohyanagi et al. (2018) only found an increase in $\left[\mathrm{HbO}_{2}\right]$ between 3 and 6 min during a 20min moderate-intensity cycling exercise, after which $\left[\mathrm{HbO}_{2}\right]$ stabilized to completion. Interestingly, Ohyanagi et al. (2018) and Tsubaki et al. (2018) both measured skin blood flow and reported that skin blood flow increased over the time of the exercise and decreased again during the postexercise rest, supporting the results of Miyazawa et al. (2013).

A gradual increase in $\left[\mathrm{HbO}_{2}\right]$ over time is present both at the light and moderate intensities and supports the results of the abovementioned incremental exercise research. In MIT, prefrontal- $\mathrm{HbO}_{2}$ levels increase to RCP, which persist in above moderate intensities. This indicates that at light-intensity and moderate-intensity exercise, PFC oxygenation increases gradually until a steady state is reached. However, it is not clear whether the increased prefrontal oxygenation is the result of an increase in cerebral $\mathrm{O}_{2}$ metabolism and neuronal activity. An increase in skin blood flow, through elevated MAP, seems to be correlated with increases in $\left[\mathrm{HbO}_{2}\right]$. The changes measured by NIRS are the result of changes that occur throughout the volume of tissue traversed by the NIR light. The signal will therefore invariably contain a superficial interference from both emitter and receiver optodes. The inclusion of short-separation channels into the NIRS optode template could reduce superficial contamination through skin blood flow, which is influenceable by systemic changes (Gagnon et al., 2014). Gagnon et al. (2014) found a $33 \%$ reduction in signal noise for $\mathrm{HbO}_{2}$ with the inclusion of one short channel and a $59 \%$ noise reduction when two short channels, one at the emitter and one at the detector, in comparison with the standard method. The role of an increased cerebral $\mathrm{O}_{2}$ metabolism through neuronal activity can, however, not be ruled out. Suzuki et al. (2004) stated that PFC might be involved in controlling locomotion to adapt to the increasing speed in the acceleration phases during walking and running. Before the start of locomotion, an increase in $\left[\mathrm{HbO}_{2}\right]$ and [tHb] was found, especially during the $9 \mathrm{~km} / \mathrm{h}$ trial, indicating a neuronal activation at PFC.

\section{Vigorous Intensity}

Two studies investigated the effects of vigorous cycling exercise on PFC oxygenation (Rupp et al., 2013; Giles et al., 2014). The results showed that prefrontal oxygenation $\left[\mathrm{HbO}_{2}\right]$ increased until reaching a steady state, which is similar during light-tomoderate intensities. Vigorous intensity ends at the verge of RCP but is still located below this threshold. In Rupp et al. (2013) and Giles et al. (2014), HHb remained unaffected during the first few minutes of exercise after which it progressively increases. Interestingly, both $\mathrm{HbO}_{2}$ and $\mathrm{tHb}$ start to increase from the onset of an endurance task. However, $\mathrm{CBF}$ is not directly measured but can be interpreted as an estimate of tHb and $\mathrm{HbO}_{2}$. This illustrates that there is an increase in $\mathrm{O}_{2}$ supply to the PFC during the initiation of vigorous-intensity exercise. The continuous gradual increase of $\mathrm{HbO}_{2}, \mathrm{tHb}$, and, therefore, $\mathrm{CBF}$, during $84 \%$
HRmax (vigorous intensity), is likely the result of RCP occurring between 85 and 91\% HRmax. Given that the targeted workload of $84 \%$ HRmax is below this range, it may have resulted in being insufficient to meet RCP and thus a decrease in $\mathrm{HbO}_{2}$ and $\mathrm{tHb}$. This confirms our hypothesis that PFC increases during prolonged submaximal exercise until a steady state is reached.

\section{Near-to-Maximal Intensity}

Six studies assessed prefrontal oxygenation at near-to-maximal intensities. Incremental exercise studies have suggested that PFC oxygenation cannot be maintained and occurs in PFC. The tipping point of this deoxygenating phase is RCP. Five studies confirm this hypothesis during both running and cycling exercises. Billaut et al. (2010) and Pires et al. (2016) showed that prefrontal oxygenation could not be maintained throughout the trial, whereas in Santos-Concejero et al. (2015) $\mathrm{HbO}_{2}$ and $\mathrm{tHb}$ increased from $\mathrm{km} 2.5$ until the end. Despite the presence of identical TT running trials, Santos-Concejero et al. (2015) included elite Kenyan runners who underwent prenatal exposure to high altitude and high physical activity levels during childhood, which may have facilitated the maintenance of cerebral oxygenation. Prefrontal deoxygenation shown in Billaut et al. (2010) and Pires et al. (2016) did not result in a decrease in the motor output. These results may indicate that the duration of the TT task is insufficient for PFC oxygenation to influence the motor output and that deoxygenation remains within a range that does not hinder strenuous performance. However, these findings do not discount the suggestion that pacing strategies may influence the cerebral function metabolic status. Moreover, an initial increase in blood flow along with $\mathrm{HbO}_{2}$ and $\mathrm{HHb}$ was thought to be the result of an increase in cardiac output. Because the near-to-maximal intensities exceed RCP, cerebral vasoconstriction could have led to prefrontal deoxygenation. This statement could, however, be questioned as to the deoxygenation in Santos-Concejero et al. (2015) was characterized by an increase in $\mathrm{tHb}$ through elevated $\mathrm{HHb}$ levels and a decrease in $\mathrm{HbO}_{2}$. Similarly, Santos-Concejero et al. (2017) found that during a fatigue training test, $\triangle\left[\mathrm{HbO}_{2}\right]$ that was measured at the end of each running repetition was decreased over the course of the trial. The decrease in $\mathrm{HbO}_{2}$ occurred simultaneously with increases in $\mathrm{HHb}$ over the course of the session with increases during each running bout, and a subsequent decrease during each 30-s recovery period until the end. It was stated that the speed at which participants performed was significantly faster than their expected speed at RCP, which is supported by a high lactate observation. A major limitation to this study, however, is that $\mathrm{tHb}$, an indicator for CBF, is not measured in this study. Therefore, it is likely that hyperventilation-induced hypocapnia after RCP leads to deoxygenation. This study also highlights that high-intensity interval training (HIIT) results in a decrease in prefrontal oxygenation. In relation to prolonged endurance exercise, this could suggest that sequential intermediate near-tomaximal intensity efforts (e.g., repeated breakaways in cycling races) can result in a decrease in prefrontal oxygenation.

Regarding supramaximal exercise, Shibuya et al. (2004) examined TTE performance at $120 \%$ and showed that $\left[\mathrm{HbO}_{2}\right]$ increased during the first $30 \mathrm{~s}$, after which $\left[\mathrm{HbO}_{2}\right]$ and $[\mathrm{tHb}]$ 
gradually decreased and were significantly lower than preexercise. $[\mathrm{HHb}$ ] remained unchanged throughout the trial. The initial increase in $\left[\mathrm{HbO}_{2}\right]$ and $[\mathrm{tHb}]$ is likely to be the result of a sudden increase in blood pressure through the start of locomotion against high resistance. On the other hand, the decrease in $\left[\mathrm{HbO}_{2}\right]$ and $[\mathrm{tHb}]$ can be linked with increasing end-tidal $\mathrm{CO}_{2}$ concentrations, which result in cerebral vasoconstriction through hyperventilation, and in turn, lead to a decrease in $\mathrm{CBF}$ and thus in tHb and $\mathrm{HbO}_{2}$. Shibuya et al. (2004) proposed a potential role for cerebral fatigue as a result of the changes in oxygenation. However, no study has provided evidence to support this hypothesis. This is the only study that has assessed PFC oxygenation during supramaximal exercise, indicating a need for further research measuring cerebral oxygenation during prolonged supramaximal exercise.

Radel et al. (2017) investigated the hemodynamic changes that arise when coping with the expectation of prolonged exercise and found that $\left[\mathrm{HbO}_{2}\right]$ increased over a 10 -min cycling task, which is in accordance with the study of Rooks et al. (2010). When participants were expected to cycle for $60 \mathrm{~min}$, the authors reported that $\left[\mathrm{HbO}_{2}\right]$ elevations at the right dorsolateral PFC were diminished and $\left[\mathrm{HbO}_{2}\right]$ elevations at the right dorsomedial PFC were higher. No differences in RPE between 10 and 60 min were found. The dorsolateral PFC is a representative region for the central executive network, whereas the dorsomedial PFC is a representative region for the default mode network (Fox et al., 2009). This highlights that the brain attempts to save mental resources by providing less activation of brain regions (dorsal PFC) associated with mental effort (the central executive network) and more toward those related to the resting activity (the default mode network) (Shibuya, 2011; Perrey and Besson, 2018). This study also indicates that psychological factors influence PFC oxygenation, suggesting that psychological interventions could positively influence PFC oxygenation and in turn improve endurance performance. Further, nutrition has also been shown to have a facilitating effect on PFC oxygenation. Decroix et al. (2018) reported an elevation in prefrontal oxygenation without having an impact on muscular oxygenation during a 20-min steady-state cycling exercise at $45 \% \mathrm{VO}_{2 \max }$ (moderate) after the supplementation of cocoa flavanols. Given that mental fatigue negatively alters endurance performance (Marcora et al., 2009; Martin et al., 2018; Pageaux and Lepers, 2018), these findings are therefore an important factor for an examination within mental fatigue and highlight that PFC is involved in proactive behavior and goal-directed exercises (Pires et al., 2018).

\section{Respiratory Compensation Point as a Cerebral Oxygenation Threshold}

Research on PFC oxygenation within dynamic whole-body endurance performance throughout the entire spectrum of intensities (low, moderate, vigorous, near-to-maximal, and supramaximal intensities) is in accordance with research examining maximal incremental exercise. Our systematic review has found strong evidence that RCP is an important ThCox. Collectively, research indicates that PFC oxygenation increases throughout intensities below the RCP threshold and reaches a steady state during continuous sub-RCP workload tasks. However, during prolonged exercise at the intensities that exceed RCP, the increase or preservation of prefrontal $\mathrm{HbO}_{2}$ cannot be maintained and the deoxygenation of PFC takes place. The findings presented in this review show no evidence that an increase in cerebral energy metabolism is responsible for the deoxygenation of PFC and limiting factors of endurance performance. When RCP is reached during exercise, an increase in respiratory ventilation results in hypocapnia. Cerebral autoregulation reacts to hypocapnia by causing cerebral vasoconstriction, resulting in a decrease in $\mathrm{CBF}$ and thus in $[\mathrm{tHb}]$, $\left[\mathrm{HbO}_{2}\right]$, and [HHb] (Ogoh and Ainslie, 2009).

\section{Limitations and Future Research}

Although this systematic review has provided novel insights into the influence of PFC oxygenation on whole-body endurance exercise, there are some limitations that need to be considered. Firstly, there is a need for a consensus and transparency in reporting the results within whole-body endurance exercise research. RCP acts as a tipping point/threshold for PFC oxygenation. However, this threshold was not consistently determined or referenced in every study. To ensure the adequate comparison between studies and to classify exercise intensity, we calculated $\mathrm{VO}_{2}$ and $\%$ of $\mathrm{VO}_{2 \max }$ of the described datapoints and the metabolic equivalent of task (MET). To facilitate a comparison between studies in future research, each threshold or discussed data point should be referred to as a percentage of $\mathrm{VO}_{2 \mathrm{max} / \text { peak }}$. Furthermore, concerning the reporting of the results, the two factors that are overlooked in most of the included studies are MAP and the training status of the subjects. MAP is closely related to oxygenation and (cerebral) blood flow and can, therefore, give interesting insights into the discussion of the results. However, recently, Castle-Kirszbaum et al. (2021) released a systematic review on a cardio-cerebral coupling and indicated that the current literature is insufficiently robust to confirm an independent relationship between the cardiac output and CBF. Regarding the training status, future research within this field should address participant characteristics related to the performance level as described by De Pauw et al. (2013) and Decroix et al. (2016) for male and female subjects, respectively. Secondly, future research should include objective measures for CBF (e.g., PET and TCD) simultaneously with NIRS to confirm the hypothesis that exceeding RCP results in cerebral vasoconstriction that in turn decreases PFC oxygenation. Moreover, a short-separation channel $(8 \mathrm{~mm})$ (Herold et al., 2018) needs to be included within the NIRS setup to determine whether changes in cerebral oxygenation are not the result of changes in local skin blood flow. However, there is a lack of consensus for the fNIRS application (optode placement, IOD, etc.), which complicates comparisons between studies (see Herold et al., 2018 for guidelines).

In this systematic review, only 6 of 28 studies assessed laterality. In future research, PFC laterality is an important factor given that the left and right PFC play different roles and may influence decision-making during endurance exercise. However, given a low number of studies to assess laterality, we believe 
that it was inappropriate to make comparisons between all the studies. Future research should, therefore, endeavor to examine PFC laterality to provide a greater understanding of how this may influence endurance performance. Another limitation is that the environmental conditions were rarely mentioned. Given that ambient temperature and relative humidity influence endurance performance and can facilitate vasoconstriction or vasodilatation, it is likely that these could influence NIRS parameters. Further research should examine if the deoxygenation of PFC determines whether one stops or prolongs during an endurance task and if the interventions that postpone PFC deoxygenation could result in improved performances. Recently, the first study on this subject has been published (Dallaway et al., 2021). The authors reported that brain endurance training resulted in improved performance on muscular endurance handgrip tasks, which might occur with higher prefrontal oxygenation. This traininginduced increase in prefrontal oxygenation was accompanied by a reduced mental effort during the physical task, making oxygenation a potential countermeasure for mental fatigue. These preliminary data are promising, and a need exists for more research to replicate this study design on whole-body endurance exercise tasks.

\section{CONCLUSION}

To conclude, this systematic review provides a detailed overview of how cerebral oxygenation at PFC reacts to various exercise intensities. As hypothesized, we found that PFC oxygenation increases at low, moderate, and vigorous intensities and decreases at near-to-maximal and supramaximal intensities. Moreover, a steady state could be reached at the intensities below RCP. RCP can also be identified as an important ThCox given that PFC oxygenation cannot be maintained and decreases until the cessation of whole-body endurance exercise at this point. The proposed mechanism behind this is that through an increase

\section{REFERENCES}

Ainslie, P. N., Barach, A., Murrell, C., Hamlin, M., Hellemans, J., and Ogoh, S. (2007). Alterations in cerebral autoregulation and cerebral blood flow velocity during acute hypoxia: rest and exercise. Am. J. Physiol. Heart Circ. Physiol. 292, H976-H983. doi: 10.1152/ajpheart.00639.2006

Amann, M. (2011). Central and peripheral fatigue: interaction during cycling exercise in humans. Med. Sci. Sports Exerc. 43, 2039-2045. doi: 10.1249/MSS. 0b013e31821f59ab

Amann, M., Hopkins, W. G., and Marcora, S. M. (2008). Similar sensitivity of time to exhaustion and time-trial time to changes in endurance. Med. Sci. Sports Exer. 40, 574-578. doi: 10.1249/MSS.0b013e31815e728f

Angius, L., Santarnecchi, E., Pascual-Leone, A., and Marcora, S. M. (2019). Transcranial direct current stimulation over the left dorsolateral prefrontal cortex improves inhibitory control and endurance performance in healthy individuals. Neuroscience 419, 34-45. doi: 10.1016/j.neuroscience.2019.08.052

Asahara, R., and Matsukawa, K. (2018). Decreased prefrontal oxygenation elicited by stimulation of limb mechanosensitive afferents during cycling exercise. Am. J. Physiol. Regul. Integr. Comp. Physiol. 315, R230-R240. doi: 10.1152/ajpregu.00454.2017

Bhambhani, Y., Malik, R., and Mookerjee, S. (2007). Cerebral oxygenation declines at exercise intensities above the respiratory compensation threshold. Respir. Physiol. Neurobiol. 156, 196-202. doi: 10.1016/j.resp.2006.08.009 in ventilation, as a response to maintain the acid-base balance after exceeding RCP leads to cerebral vasoconstriction and therefore also in cerebral oxygenation. These findings reinforce and expand the knowledge on cerebral oxygenation during whole-body endurance exercise. Future research should examine whether maintaining/improving PFC oxygenation can improve endurance performance.

\section{DATA AVAILABILITY STATEMENT}

The original contributions presented in the study are included in the article/supplementary material, further inquiries can be directed to the corresponding author/s.

\section{AUTHOR CONTRIBUTIONS}

JDW performed the design of the search strategy. Subsequently, MP, JVC, JH, JD-G, and BR revised the design. JDW and JH did screening on title and abstract, while JDW, MP, JH, JVC, and BR conducted full text screening. JDW, MP, and JH first conducted the data analysis. JVC and BR later revised and updated the data analysis. JDW and JH performed quality assessment and also designed the quality assessment table together with MP. MV and JDW performed calculations for exercise intensity and performance level. JDW wrote the first draft of the manuscript, which was later altered by MP, JH, MV, PH, JD-G, JVC, BR, and RM. All authors read, revised, and approved the final manuscript.

\section{ACKNOWLEDGMENTS}

We would like to thank the Luxemburg Institute of Research in Orthopedics, Sport Medicine and Science (LIROMS) for their valuable contribution to this work.

Billaut, F., Davis, J. M., Smith, K. J., Marino, F. E., and Noakes, T. D. (2010). Cerebral oxygenation decreases but does not impair performance during selfpaced, strenuous exercise. Acta Physiol. 198, 477-486. doi: 10.1111/j.1748-1716. 2009.02058.x

Borg, G. (1998). Borg's Perceived exertion and pain scales. Champaign, IL: Human Kinetics.

Castle-Kirszbaum, M., Parkin, W. G., Goldschlager, T., and Lewis, P. M. (2021). Cardiac output and cerebral blood flow: A systematic review of cardiocerebral coupling. J. Neurosurg. Anesthesiol. 2021:768. doi: 10.1097/ANA. 0000000000000768

Dallaway, N., Lucas, S. J. E., and Ring, C. (2021). Concurrent brain endurance training improves endurance exercise performance. J. Sci. Med. Sport 24, 405-411. doi: 10.1016/j.jsams.2020.10.008

Decroix, L., de Pauw, K., Foster, C., and Meeusen, R. (2016). Guidelines to classify female subject groups in sport-science research. Int. J. Sports Physiol. Perform. 11, 204-213. doi: 10.1123/ijspp.2015-0153

Decroix, L., Tonoli, C., Lespagnol, E., Balestra, C., Descat, A., Drittij-Reijnders, M. J., et al. (2018). One-week cocoa flavanol intake increases prefrontal cortex oxygenation at rest and during moderate-intensity exercise in normoxia and hypoxia. J. Appl. Physiol. 125, 8-18. doi: 10.1152/japplphysiol.00055.2018

De Pauw, K., Roelands, B., Cheung, S. S., de Geus, B., Rietjens, G., and Meeusen, R. (2013). Guidelines to classify subject groups in sport-science research. Int. J. Sports Physiol. Perform. 8, 111-122. doi: 10.1123/ijspp.8.2.111 
Ekkekakis, P. (2009). Illuminating the black box: investigating prefrontal cortical hemodynamics during exercise with near-infrared spectroscopy. J. Sport Exerc. Psychol. 31, 505-553. doi: 10.1123/jsep.31.4.505

Fox, M. D., Zhang, D., Snyder, A. Z., and Raichle, M. E. (2009). The global signal and observed anticorrelated resting state brain networks. J. Neurophysiol. 101, 3270-3283. doi: 10.1152/jn. 90777.2008

Fumoto, M., Oshima, T., Kamiya, K., Kikuchi, H., Seki, Y., Nakatani, Y., et al. (2010). Ventral prefrontal cortex and serotonergic system activation during pedaling exercise induces negative mood improvement and increased alpha band in EEG. Behav. Brain Res. 213, 1-9. doi: 10.1016/j.bbr.2010. 04.017

Gagnon, L., Yücel, M. A., Boas, D. A., and Cooper, R. J. (2014). Further improvement in reducing superficial contamination in NIRS using double short separation measurements. NeuroImage 85, 127-135. doi: 10.1016/j. neuroimage.2013.01.073

Galán-Rioja, M., González-Mohíno, F., Poole, D. C., and González-Ravé, J. M. (2020). Relative proximity of critical power and metabolic/ventilatory thresholds: systematic review and meta-analysis. Sports Med. 50, 1771-1783. doi: $10.1007 / s 40279-020-01314-8$

Garber, C. E., Blissmer, B., Deschenes, M. R., Franklin, B. A., Lamonte, M. J., Lee, I. M., et al. (2011). American college of sports medicine position stand. Quantity and quality of exercise for developing and maintaining cardiorespiratory, musculoskeletal, and neuromotor fitness in apparently healthy adults: guidance for prescribing exercise. Med. Sci. Sports Exerc. 43, 1334-1359. doi: 10.1249/ MSS.0b013e318213fefb

Gastin, P. B. (2001). Energy system interaction and relative contribution during maximal exercise. Sports Med. 31, 725-741. doi: 10.2165/00007256-20013110000003

Giles, G. E., Brunyé, T. T., Eddy, M. D., Mahoney, C. R., Gagnon, S. A., Taylor, H. A., et al. (2014). Acute exercise increases oxygenated and deoxygenated hemoglobin in the prefrontal cortex. Neuroreport 25, 1320-1325. doi: 10.1097/ WNR.0000000000000266

Girard, O., and Racinais, S. (2014). Combining heat stress and moderate hypoxia reduces cycling time to exhaustion without modifying neuromuscular fatigue characteristics. Eur. J. Appl. Physiol. 114, 1521-1532. doi: 10.1007/s00421-0142883-0

Glover, G. H. (2011). Overview of functional magnetic resonance imaging. Neurosur. Clin. N. Am. 22:133. doi: 10.1016/j.nec.2010.11.001

Herold, F., Wiegel, P., Scholkmann, F., and Muller, N. G. (2018). Applications of Functional Near-Infrared Spectroscopy (fNIRS) neuroimaging in exercisecognition science: A systematic, methodology-focused review. J. Clin. Med. 7:466. doi: $10.3390 / \mathrm{jcm} 7120466$

Hiura, M., Nariai, T., Takahashi, K., Muta, A., Sakata, M., Ishibashi, K., et al. (2018). Dynamic exercise elicits dissociated changes between tissue oxygenation and cerebral blood flow in the prefrontal cortex: A study using NIRS and PET. Adv. Exp. Med. Biol. 1072, 269-274. doi: 10.1007/978-3-319-91287-5_43

Ichinose, Y., Morishita, S., Suzuki, R., Endo, G., and Tsubaki, A. (2020). Comparison of the effects of continuous and intermittent exercise on cerebral oxygenation and cognitive function. Adv. Exp. Med. Biol. 1232, 209-214. doi: 10.1007/978-3-030-34461-0_26

Ide, K., and Secher, N. H. (2000). Cerebral blood flow and metabolism during exercise. Prog. Neurobiol. 61, 397-414. doi: 10.1016/S0301-0082(99)00057-X

Ide, K., Horn, A., and Secher, N. H. (1999). Cerebral metabolic response to submaximal exercise. J. Appl. Physiol. 87, 1604-1608. doi: 10.1152/jappl.1999. 87.5.1604

Jung, R., Moser, M., Baucsek, S., Dern, S., and Schneider, S. (2015). Activation patterns of different brain areas during incremental exercise measured by nearinfrared spectroscopy. Exp. Brain Res. 233, 1175-1180. doi: 10.1007/s00221015-4201-4

Kenney, W. L., Wilmore, J. H., and Costill, D. L. (2012). Physiology of sport and exercise, 5th Edn. Champaign, IL: Human Kinetics Publishers.

Keramidas, M. E., Kounalakis, S. N., Eiken, O., and Mekjavic, I. B. (2011). Muscle and cerebral oxygenation during exercise performance after short-term respiratory work. Respir. Physiol. Neurobiol. 175, 247-254. doi: 10.1016/j.resp. 2010.11.009

Kmet, L., and Lee, R. (2004). Standard Quality Assessment Criteria for Evaluating Primary Research Papers from a Variety of Fields AHFMRHTA Initiative20040213. HTA Initiative, 2.
Kojima, S., Morishita, S., Hotta, K., Qin, W., Kato, T., Oyama, K., et al. (2021). Relationship between decrease of oxygenation during incremental exercise and partial pressure end-tidal carbon dioxide: near-infrared spectroscopy vector analysis. Adv. Exp. Med. Biol. 1269, 119-124. doi: 10.1007/978-3-030-48238$1 \_19$

Kojima, S., Morishita, S., Qin, W., and Tsubaki, A. (2020). Cerebral oxygenation dynamics of the prefrontal cortex and motor-related area during cardiopulmonary exercise test: A near-infrared spectroscopy study. Adv. Exp. Med. Biol. 1232, 231-237. doi: 10.1007/978-3-030-34461-0_29

Kounalakis, S. N., and Geladas, N. D. (2012). Cardiovascular drift and cerebral and muscle tissue oxygenation during prolonged cycling at different pedalling cadences. Appl. Physiol. Nutr. Metab. 37, 407-417. doi: 10.1139/h2012-011

Lassen, N. A. (1959). Cerebral blood flow and oxygen consumption in man. Physiol. Rev. 39, 183-238. doi: 10.1152/physrev.1959.39.2.183

Leff, D. R., Orihuela-Espina, F., Elwell, C. E., Athanasiou, T., Delpy, D. T., Darzi, A. W., et al. (2011). Assessment of the cerebral cortex during motor task behaviours in adults: a systematic review of functional near infrared spectroscopy (fNIRS) studies. Neuroimage 54, 2922-2936. doi: 10.1016/j. neuroimage.2010.10.058

Leon-Carrion, J., and Leon-Dominguez, U. (2012). Functional Near-Infrared Spectroscopy (fNIRS): Principles and Neuroscientific Applications. London: IntechOpen. doi: 10.5772/23146

Liberati, A., Altman, D. G., Tetzlaff, J., Mulrow, C., Gøtzsche, P. C., Ioannidis, J. P., et al. (2009). The PRISMA statement for reporting systematic reviews and metaanalyses of studies that evaluate health care interventions: explanation and elaboration. J. Clin. Epidemiol. 62, e1-e34. doi: 10.1016/j.jclinepi.2009.06.006

Marcora, S. M., Staiano, W., and Manning, V. (2009). Mental fatigue impairs physical performance in humans. J. Appl. Physiol. (1985) 106, 857-864. doi: 10.1152/japplphysiol.91324.2008

Martin, K., Meeusen, R., Thompson, K. G., Keegan, R., and Rattray, B. (2018). Mental fatigue impairs endurance performance: A physiological explanation. Sports Med. 48, 2041-2051. doi: 10.1007/s40279-018-0946-9

Matsukawa, K., Ishii, K., Liang, N., Endo, K., Ohtani, R., Nakamoto, T., et al. (2015). Increased oxygenation of the cerebral prefrontal cortex prior to the onset of voluntary exercise in humans. J. Appl. Physiol. (1985) 119, 452-462. doi: 10.1152/japplphysiol.00406.2015

McArdle, W. D., Katch, F. I., and Katch, V. L. (2010). Exercise physiology: nutrition, energy and human performance. Philadelphia: Lippincott Williams \& Wilkins.

Mccormick, A., Meijen, C., and Marcora, S. (2015). Psychological determinants of whole-body endurance performance. Sports Med. 45, 997-1015. doi: 10.1007/ s40279-015-0319-6

Miyazawa, T., Horiuchi, M., Komine, H., Sugawara, J., Fadel, P. J., and Ogoh, S. (2013). Skin blood flow influences cerebral oxygenation measured by nearinfrared spectroscopy during dynamic exercise. Eur. J. Appl. Physiol. 113, 2841-2848. doi: 10.1007/s00421-013-2723-7

Nielsen, H. B., Boushel, R., Madsen, P., and Secher, N. H. (1999). Cerebral desaturation during exercise reversed by $\mathrm{O} 2$ supplementation. Am. J. Physiol. 277, H1045-H1052. doi: 10.1152/ajpheart.1999.277.3.H1045

Nybo, L., and Rasmussen, P. (2007). Inadequate cerebral oxygen delivery and central fatigue during strenuous exercise. Exerc. Sport Sci. Rev. 35, 110-118. doi: 10.1097/jes.0b013e3180a031ec

Ogoh, S., and Ainslie, P. N. (2009). Cerebral blood flow during exercise: mechanisms of regulation. J. Appl. Physiol. 107, 1370-1380. doi: 10.1152/ japplphysiol.00573.2009

Ohyanagi, H., Tsubaki, A., Morishita, S., Obata, H., Qin, W., and Onishi, H. (2018). Changes in the prefrontal cortex oxygenation levels during cycling in the supine and upright positions. Adv. Exp. Med. Biol. 1072, 133-137. doi: 10.1007/978-3-319-91287-5_21

Oussaidene, K., Prieur, F., Tagougui, S., Abaidia, A., Matran, R., and Mucci, P. (2015). Aerobic fitness influences cerebral oxygenation response to maximal exercise in healthy subjects. Respir. Physiol. Neurobiol. 205, 53-60. doi: 10.1016/ j.resp.2014.10.009

Ouzzani, M., Hammady, H., Fedorowicz, Z., and Elmagarmid, A. (2016). Rayyana web and mobile app for systematic reviews. Syst. Rev. 5:210. doi: 10.1186/ s13643-016-0384-4

Page, M. J., Mckenzie, J. E., Bossuyt, P. M., Boutron, I., Hoffmann, T. C., Mulrow, C. D., et al. (2021). The PRISMA 2020 statement: An updated guideline for reporting systematic reviews. BMJ 372:n71. doi: 10.1136/bmj.n71 
Pageaux, B., and Lepers, R. (2018). The effects of mental fatigue on sportrelated performance. Prog. Brain Res. 240, 291-315. doi: 10.1016/bs.pbr.2018. 10.004

Pageaux, B., Marcora, S. M., and Lepers, R. (2013). Prolonged mental exertion does not alter neuromuscular function of the knee extensors. Med. Sci. Sports Exerc. 45, 2254-2264. doi: 10.1249/MSS.0b013e31829b504a

Périard, J., and Racinais, S. (2015). Performance and pacing during cycle exercise in hyperthermic and hypoxic conditions. Med. Sci. Sports Exerc. 48:1. doi: 10.1249/MSS.0000000000000839

Perrey, S., and Besson, P. (2018). Studying brain activity in sports performance: Contributions and issues. Prog. Brain Res. 240, 247-267. doi: 10.1016/bs.pbr. 2018.07.004

Pires, F. O., Dos Anjos, C. A., Covolan, R. J., Pinheiro, F. A., St Clair Gibson, A., Noakes, T. D., et al. (2016). Cerebral regulation in different maximal aerobic exercise modes. Front. Physiol. 7:253. doi: 10.3389/fphys.2016.00253

Pires, F. O., Silva-Júnior, F. L., Brietzke, C., Franco-Alvarenga, P. E., Pinheiro, F. A., França, N. M., et al. (2018). Mental fatigue alters cortical activation and psychological responses, impairing performance in a distance-based cycling trial. Front. Physiol. 9:227. doi: 10.3389/fphys.2018.00227

Poulin, M. J., Liang, P. J., and Robbins, P. A. (1996). Dynamics of the cerebral blood flow response to step changes in end-tidal PCO2 and PO2 in humans. J. Appl. Physiol. 81, 1084-1095. doi: 10.1152/jappl.1996.81.3.1084

Racinais, S., and Girard, O. (2012). Neuromuscular failure is unlikely to explain the early exercise cessation in hot ambient conditions. Psychophysiology 49, 853-865. doi: 10.1111/j.1469-8986.2012.01360.x

Radel, R., Brisswalter, J., and Perrey, S. (2017). Saving mental effort to maintain physical effort: a shift of activity within the prefrontal cortex in anticipation of prolonged exercise. Cogn. Affect. Behav. Neurosci. 17, 305-314. doi: 10.3758/ s13415-016-0480-x

Rausch, S. M., Whipp, B. J., Wasserman, K., and Huszczuk, A. (1991). Role of the carotid bodies in the respiratory compensation for the metabolic acidosis of exercise in humans. J. Physiol. 444, 567-578. doi: 10.1113/jphysiol.1991. sp018894

Robertson, C. V., and Marino, F. E. (2016). A role for the prefrontal cortex in exercise tolerance and termination. J. Appl. Physiol. 120, 464-466. doi: 10.1152/ japplphysiol.00363.2015

Rooks, C., Thom, N., Mccully, K., and Dishman, R. (2010). Effects of incremental exercise on cerebral oxygenation measured by near-infrared spectroscopy: A systematic review. Prog. Neurobiol. 92, 134-150. doi: 10.1016/j.pneurobio.2010. 06.002

Rupp, T., and Perrey, S. (2008). Prefrontal cortex oxygenation and neuromuscular responses to exhaustive exercise. Eur. J. Appl. Physiol. 102, 153-163. doi: 10. 1007/s00421-007-0568-7

Rupp, T., Jubeau, M., Millet, G. Y., Wuyam, B., Levy, P., Verges, S., et al. (2013). Muscle, prefrontal, and motor cortex oxygenation profiles during prolonged fatiguing exercise. Adv. Exp. Med. Biol. 789, 149-155. doi: 10.1007/978-1-46147411-1_21

Santos-Concejero, J., Billaut, F., Grobler, L., Oliván, J., Noakes, T. D., and Tucker, R. (2015). Maintained cerebral oxygenation during maximal self-paced exercise in elite Kenyan runners. J. Appl. Physiol. (1985) 118, 156-162. doi: 10.1152/ japplphysiol.00909.2014

Santos-Concejero, J., Billaut, F., Grobler, L., Oliván, J., Noakes, T. D., and Tucker, R. (2017). Brain oxygenation declines in elite Kenyan runners during a maximal interval training session. Eur. J. Appl. Physiol. 117, 1017-1024. doi: 10.1007/ s00421-017-3590-4

Scarapicchia, V., Brown, C., Mayo, C., and Gawryluk, J. R. (2017). Functional magnetic resonance imaging and functional near-infrared spectroscopy: insights from combined recording studies. Front. Hum. Neurosci. 11:419. doi: 10.3389/fnhum.2017.00419

Shibuya, K. (2011). The activity of the primary motor cortex ipsilateral to the exercising hand decreases during repetitive handgrip exercise. Physiol. Meas. 32, 1929-1939. doi: 10.1088/0967-3334/32/12/004

Shibuya, K., Tanaka, J., Kuboyama, N., Murai, S., and Ogaki, T. (2004). Cerebral cortex activity during supramaximal exhaustive exercise. J. Sports Med. Phys. Fitness 44, 215-219.

Stevens, D., Halaki, M., Chow, C. M., and O’Dwyer, N. (2018). The effects of multi-stage exercise with and without concurrent cognitive performance on cardiorespiratory and cerebral haemodynamic responses. Eur. J. Appl. Physiol. 118, 2121-2132. doi: 10.1007/s00421-018-3942-8
Subudhi, A. W., Dimmen, A. C., and Roach, R. C. (2007). Effects of acute hypoxia on cerebral and muscle oxygenation during incremental exercise. J. Appl. Physiol. 103, 177-183. doi: 10.1152/japplphysiol.01460.2006

Suzuki, M., Miyai, I., Ono, T., Oda, I., Konishi, I., Kochiyama, T., et al. (2004). Prefrontal and premotor cortices are involved in adapting walking and running speed on the treadmill: an optical imaging study. Neuroimage 23, 1020-1026. doi: 10.1016/j.neuroimage.2004.07.002

Swain, D. P. (2000). Energy cost calculations for exercise prescription: An update. Sports Med. 30, 17-22. doi: 10.2165/00007256-200030010-00002

Takehara, N., Tsubaki, A., Yamazaki, Y., Kanaya, C., Sato, D., Morishita, S., et al. (2017). Changes in oxyhemoglobin concentration in the prefrontal cortex and primary motor cortex during low- and moderate-intensity exercise on a cycle ergometer. Adv. Exp. Med. Biol. 977, 241-247. doi: 10.1007/978-3-319-55231$6 \_33$

Tempest, G. D., Eston, R. G., and Parfitt, G. (2014). Prefrontal cortex haemodynamics and affective responses during exercise: a multi-channel near infrared spectroscopy study. PLoS One 9:e95924. doi: 10.1371/journal.pone. 0095924

Tempest, G. D., Eston, R. G., and Parfitt, G. (2017). A comparison of head motion and prefrontal haemodynamics during upright and recumbent cycling exercise. Clin. Physiol. Funct. Imag. 37, 723-729. doi: 10.1111/cpf.12365

Tempest, G., and Parfitt, G. (2016). Self-reported tolerance influences prefrontal cortex hemodynamics and affective responses. Cogn. Affect. Behav. Neurosci. 16, 63-71. doi: 10.3758/s13415-015-0374-3

Timinkul, A., Kato, M., Omori, T., Deocaris, C. C., Ito, A., Kizuka, T., et al. (2008). Enhancing effect of cerebral blood volume by mild exercise in healthy young men: a near-infrared spectroscopy study. Neurosci. Res. 61, 242-248. doi: 10.1016/j.neures.2008.03.012

Tsubaki, A., Morishita, S., Tokunaga, Y., Sato, D., Qin, W., Kojima, S., et al. (2020). Effect of exercise duration on post-exercise persistence of oxyhemoglobin changes in the premotor cortex: A near-infrared spectroscopy study in moderate-intensity cycling exercise. Adv. Exp. Med. Biol. 1232, 193-199. doi: 10.1007/978-3-030-34461-0_24

Tsubaki, A., Morishita, S., Tokunaga, Y., Sato, D., Tamaki, H., Yamazaki, Y., et al. (2018). Changes in cerebral oxyhaemoglobin levels during and after a single 20minute bout of moderate-intensity cycling. Adv. Exp. Med. Biol. 1072, 127-131. doi: 10.1007/978-3-319-91287-5_20

Tsubaki, A., Takai, H., Kojima, S., Miyaguchi, S., Sugawara, K., Sato, D., et al. (2016). Changes in cortical oxyhaemoglobin signal during low-intensity cycle ergometer activity: a near-infrared spectroscopy study. Oxygen transport to tissue XXXVII. Adv. Exper. Med. Biol. 876, 79-85. doi: 10.1007/978-1-49393023-4_10

Van Cutsem, J., Roelands, B., de Pauw, K., Meeusen, R., and Marcora, S. (2019). Subjective thermal strain impairs endurance performance in a temperate environment. Physiol. Behav. 202, 36-44. doi: 10.1016/j.physbeh.2019. 01.011

van den Aardweg, J. G., and de Groot, N. (2015). Tight coupling between inspiration and expiration after the respiratory compensation point. Eur. Respir. J. 46, A1553. doi: 10.1183/13993003.congress-2015.PA1553

Yoon, S., Zuccarello, M., and Rapoport, R. (2012). pCO2 and $\mathrm{pH}$ regulation of cerebral blood flow. Front. Physiol. 3:365. doi: 10.3389/fphys.2012.00365

Conflict of Interest: The authors declare that the research was conducted in the absence of any commercial or financial relationships that could be construed as a potential conflict of interest.

Publisher's Note: All claims expressed in this article are solely those of the authors and do not necessarily represent those of their affiliated organizations, or those of the publisher, the editors and the reviewers. Any product that may be evaluated in this article, or claim that may be made by its manufacturer, is not guaranteed or endorsed by the publisher.

Copyright (c) 2021 De Wachter, Proost, Habay, Verstraelen, Díaz-García, Hurst, Meeusen, Van Cutsem and Roelands. This is an open-access article distributed under the terms of the Creative Commons Attribution License (CC BY). The use, distribution or reproduction in other forums is permitted, provided the original author(s) and the copyright owner(s) are credited and that the original publication in this journal is cited, in accordance with accepted academic practice. No use, distribution or reproduction is permitted which does not comply with these terms. 